\title{
Linearizable Implementations Do Not Suffice for Randomized Distributed Computation
}

\author{
Wojciech Golab ${ }^{* \dagger}$ \\ HP Labs \\ wojciech.golab@hp.com
}

\author{
Lisa $\operatorname{Higham}^{\dagger}$ \\ University of Calgary \\ higham@ucalgary.ca
}

September 21, 2011

\author{
Philipp Woelfel ${ }^{\dagger}$ \\ University of Calgary \\ woelfel@ucalgary.ca
}

\begin{abstract}
Linearizability is the gold standard among algorithm designers for deducing the correctness of a distributed algorithm using implemented shared objects from the correctness of the corresponding algorithm using atomic versions of the same objects. We show that linearizability does not suffice for this purpose when processes can exploit randomization, and we discuss the existence of alternative correctness conditions. This paper makes the following contributions:

- Various examples demonstrate that using well-known linearizable implementations of objects (e.g., snapshots) in place of atomic objects can change the probability distribution of the outcomes that the adversary is able to generate. In some cases, an oblivious adversary can create a probability distribution of outcomes for an algorithm with implemented, linearizable objects, that not even a strong adversary can generate for the same algorithm with atomic objects.

- A new correctness condition for shared object implementations, called strong linearizability, is defined. We prove that a strong adversary (i.e., one that sees the outcome of each coin flip immediately) gains no additional power when atomic objects are replaced by strongly linearizable implementations. In general, no strictly weaker correctness condition suffices to ensure this. We also show that strong linearizability is a local and composable property.

- In contrast to the situation for the strong adversary, for a natural weaker adversary (one that cannot see a process' coin flip until its next operation on a shared object) we prove that there is no correspondingly general correctness condition. Specifically, any linearizable implementation of counters from atomic registers and load-linked/store-conditional objects, that satisfies a natural locality property, necessarily gives the weak adversary more power than it has with atomic counters.
\end{abstract}

${ }^{*}$ Research conducted mostly during a postdoctoral fellowship at the University of Calgary.

${ }^{\dagger}$ Research partially supported by the Natural Sciences and Engineering Research Council (NSERC) of Canada. 


\section{Introduction}

Linearizability is the gold standard among algorithm designers for deducing the correctness of a distributed algorithm using implemented shared objects from the correctness of the corresponding algorithm using atomid 1 versions of the same objects. We explore this in more detail, showing that linearizability does not suffice for this purpose when processes can exploit randomization.

In an asynchronous distributed system, processes collaborate by executing an algorithm that applies operations to a collection of shared objects. If the operations on these objects are atomic, then the result of the execution is the same as some sequential execution that could arise from an arbitrary interleaving of the processes' steps. Alternatively, some objects could be replaced by a set of software methods for the different operations on those objects. Processes would then invoke the appropriate method in order to simulate the intended atomic operation. In this case, there is a finer granularity to the interleaving of process steps. Consequently, we need to be sure that each possible result (e.g., the algorithm's return value for each process) that can arise from using the software methods could also have arisen if the operations were atomic.

This requirement is ensured if the methods provided for each object constitute a linearizable implementation [HW90] of the object. Linearizability is an especially useful and important correctness condition because it is a local property. That is, if each object in a collection of objects is replaced by its linearizable implementation, then the result of any execution that can arise from the concurrent use of the whole collection is one that could have also happened if the objects were atomic.

Linearizable implementations, however, do not preserve the probability distribution of the possible results as we transform the atomic system to the implemented one. An adversary, which schedules process steps, can "stretch out" a method call that was originally an atomic operation, and concurrently inspect the outcome of other processes' coin flips. Based on the outcomes, the scheduler can choose between alternative executions of the ongoing method call. As we will illustrate through examples, the consequences of this additional flexibility can be powerful and subtle, allowing the behaviour of the implemented system to differ dramatically from that of the atomic system. In particular, the adversary can manipulate executions so that low-probability worst-case results in the atomic system become much more probable in the implemented system.

We will see that our ability to curtail an adversary's additional power, which it can gain when atomic objects are replaced by linearizable implementations, depends in part upon the original power of the adversary. Various adversaries have been defined in literature, differing in their ability to base scheduling decisions on the random choices made by the algorithm (see Asp03 for an overview of adversary models). The main results in this paper concern two adversary models. Informally, when a process is scheduled by a strong adversary, the process executes only its next atomic operation, whether on a local or a shared object. (Coins are local objects.) When a process is scheduled by a weak adversary it executes up to and including its next step on a shared object. Thus, a strong adversary can intervene between a coin flip and the next step by the same process, whereas a weak adversary cannot. Further discussion of these adversaries, including formal definitions, appears in Section 3 .

\footnotetext{
${ }^{1}$ In this paper, an atomic operation is one that happens instantaneously, i.e., it is indivisible. But in the literature, the notion of atomicity is not used consistently. E.g., in her textbook [Lyn96], Lynch defines atomic objects to be linearizable, but Anderson and Gouda AG88, define atomicity in terms of instantaneous operations.
} 


\section{Summary of contributions}

1. Several examples demonstrate that using linearizable implemented objects in place of atomic objects in randomized algorithms allows the adversary to change the probability distribution of results. Therefore, in order to safely use implemented objects in randomized algorithms, it does not suffice to simply claim that these implementations are linearizable.

2. A new correctness condition for shared object implementations, called strong linearizability, which is strictly stronger than linearizability, is defined. We prove that a strong adversary against a randomized algorithm using strongly linearizable objects has exactly the same power as a strong adversary against the same algorithm using atomic objects. Conversely, if the set of histories that arise from a strong adversary scheduling an algorithm with implemented linearizable objects is "equivalent" to the set of histories that can arise from some strong adversary scheduling the same algorithm with atomic objects, then the former set of histories must be strongly linearizable. We also show that several known universal constructions of linearizable objects with common progress properties (e.g., wait-freedom) provide strong linearizability. Finally, we prove that strong linearizability, like linearizability, is both a local and a composable property.

3. In contrast to the situation for strong adversaries, for weak adversaries strong linearizability has no counterpart. For example, for some randomized algorithms, weak adversaries always gain additional power when strong counters (that support fetch\&inc and fetch\&dec operations) are replaced with "natural" linearizable implementations based on a set of base objects supporting reads, writes and load-linked/store-conditional operations. Consequently, to prevent weak adversaries from gaining additional power, the implementation of the counter would require additional base object types beyond what is necessary for linearizability. This result is obtained by a technically involved proof; it holds even for randomized implementations with fairly weak progress conditions (e.g., lock-freedom).

Randomization has become an important technique in the design of distributed algorithms; it allows us to circumvent some substantial impossibilities and complexity lower bounds of deterministic algorithms. Our results impact the design of randomized algorithms that use shared objects not directly supported through atomic primitives in hardware. First, simulating the required shared objects in software using "only" linearizable implementations can break the algorithm. Second, such algorithms are much easier to fix (using strong linearizability) if they are designed from the outset to work against strong adversaries, but not so if they are designed only to work against weak adversaries. Third, since there are strongly linearizable universal constructions using consensus objects, which can be implemented using compare\&swap, any system that provides compare\&swap in hardware can implement any object in a strongly linearizable way.

\section{Examples}

We begin with two examples to provide intuition and motivation, and delay the model details, which are needed for our technical results, until the next section. The examples illustrate how an adversary in a randomized algorithm gains additional power when atomic objects are replaced with implemented ones.

Atomic versus linearizable snapshots. An $n$ process snapshot object is a vector $\left(x_{1}, \ldots, x_{n}\right)$ of length $n$ that supports the atomic operations $\operatorname{UPDATE}_{p}$ and $\operatorname{SCAN}_{p}$ by any process $p \in\{1, \ldots, n\}$. 
Operation $\operatorname{UPDATE}_{p}(v)$ writes $v$ to $x_{p}$ while leaving all $x_{i}, i \neq p$ unchanged; and $\operatorname{SCAN}_{p}(v)$ returns the vector of values $\left(x_{1}, \ldots, x_{n}\right)$ to $p$.

Initialize a snapshot object for three processes to $\left(x_{p}, x_{q}, x_{r}\right)=(0,0,0)$. Suppose the processes $p, q$ and $r$ are executing the following code, and the adversary is trying to minimize the sum of the values returned in $p$ 's scan.

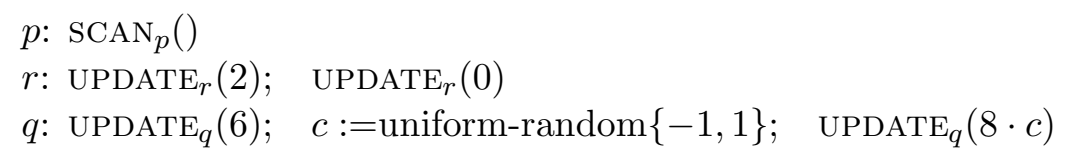

To keep the sum in $p$ 's SCAN low, the adversary can schedule either both or neither of $r$ 's UPDATE operations before $p$ 's SCAN. If the adversary is weak, the same holds for $q$ 's UPDATE operations. Thus, under the best strategy for a weak adversary, the expected value of the sum in $p$ 's SCAN is 0 . If the adversary is strong, its best strategy is to schedule $p$ 's SCAN before $q$ 's second UPDATE if $q$ 's coin flip returns 1 and after if it returns -1 . Thus, under the best strategy for a strong adversary, the expected value of the sum in $p$ 's SCAN is $(6-8) / 2=-1$.

Now suppose instead that UPDATE and SCAN are implemented from atomic registers by the well-known wait-free linearizable algorithm due to Afek, Attiya, Dolev, Gafni, Merritt and Shavit $\left[\mathrm{AAD}^{+} 93\right.$. In this algorithm, the snapshot object is implemented as an array $A[1: n]$ of registers. Let a collect denote a series of $n$ atomic reads, one for each element of $A$, in some fixed order. To perform a SCAN, each process $p$ repeatedly collects until either two successive collects are identical (a successful double collect), or $p$ observes that another process, say $r$, has executed at least two UPDATE operations to $A[r]$ during $p$ 's SCAN. In the second case, $p$ returns the last SCAN written (as we explain shortly) by $r$ during an UPDATE (a borrowed scan). To perform an UPDATE, each process $r$ must first perform a SCAN and then write the result of the SCAN together with its UPDATE argument into $A[r]$. This ensures that if a SCAN has enough failed double collects, then a borrowed SCAN is possible. With this implementation, the adversary can maneuver $p, q$ and $r$ as shown in Figure 1 .

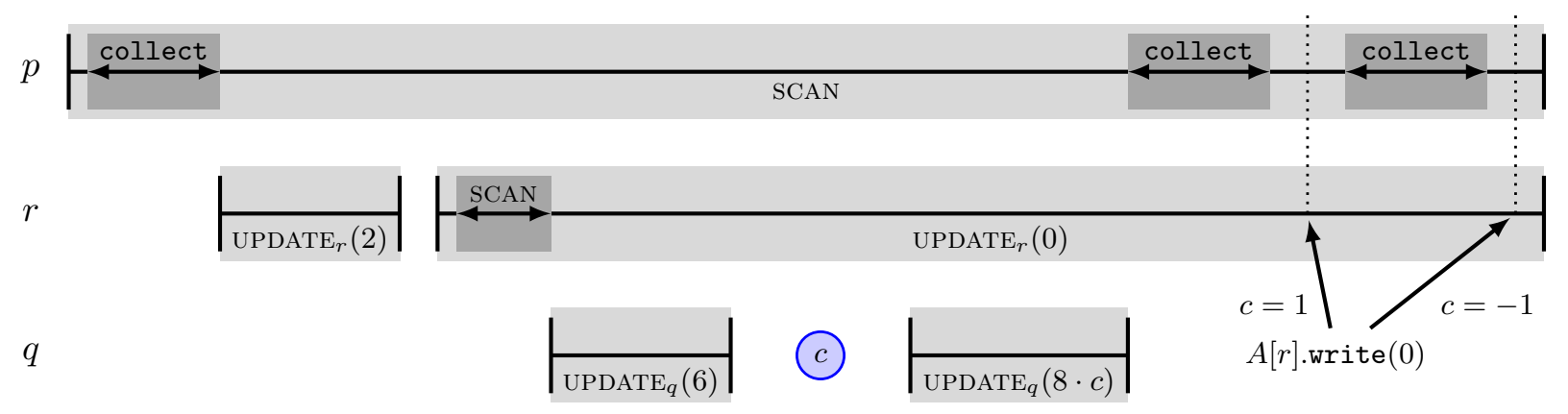

Figure 1: A "bad" scheduling using an implemented linearizable snapshot.

In this execution, $r$ applies a SCAN that returns a view $S$ with sum 2 as the first part of its second UPDATE. Then, the adversary chooses where to schedule the remainder of $r$ 's second UPDATE, which is the write to $A[r]$ of $(S, 0)$. If $q$ 's coin flip is -1 , it schedules this write after $p$ 's third collect. In this case, $p$ will have a successful double collect, which returns a view with sum $2+(-8)=-6$. If $q$ 's coin flip is 1 , the adversary schedules $r$ 's write between $p$ 's second and third collects. In 


\begin{tabular}{|c|}
\hline FunCTION WRITE $(v)$ \\
\hline $\begin{array}{l}1 A[v] . w r i t e(1) \\
2 \text { for } i=v-1, \ldots, 0 \text { do } \\
\left.3\right|_{4} A[i] . \operatorname{write}(0) \\
4 \text { end }\end{array}$ \\
\hline
\end{tabular}

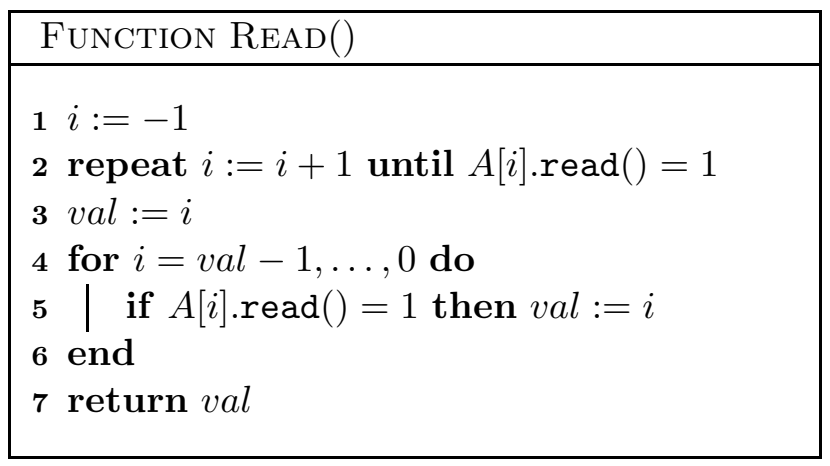

Figure 2: Linearizable implementation of multivalued SRSW registers from atomic bits.

this case, $p$ will have a failed double collect but will have seen $r$ UPDATE twice. Accordingly, $p$ borrows $r$ 's SCAN, and so $p$ 's SCAN also returns the view $S$ with sum 2 . Thus, the adversary can force an expected sum in $p$ 's SCAN of only $(-6+2) / 2=-2$. Notice, furthermore, that only a weak adversary was used to achieve this execution in the system with an implemented snapshot object.

Atomic versus linearizable registers. Since the implemented method calls give the adversary more power than it has when operations are atomic, we might conjecture that this additional power could be curtailed by appropriately restricting the adversary. The next example shows that this is not always possible.

Let $R$ denote a multi-valued atomic single-reader/single-writer (SRSW) register initialized to 1. Let processes $w$ and $p$ execute the following code:

$$
\begin{aligned}
& w: R \cdot \operatorname{WrITE}(2) ; \quad c:=\text { uniform-random }\{0,2\} ; \quad R \cdot \operatorname{WRITE}(c) \\
& p: R \cdot \operatorname{READ}()
\end{aligned}
$$

Suppose that a strong adversary is trying to minimize the value that $p$ reads. Then the adversary's best strategy is to have $p$ execute its READ either before or after both of $w$ 's WRITE operations. In either case, the expected value of $p$ 's READ is 1.

Now suppose, instead, that $R$ is implemented using Vidyasankar's linearizable implementation of single-reader/single-writer (SRSW) multivalued registers from SRSW atomic bits [Vid88. In this construction, an array $A[0 \ldots \ell]$ of SRSW binary registers is used to represent a register with domain $\{0, \ldots, \ell\}$. Value $v$ is represented by $A[v]=1$ and $A[0]=\cdots=A[v-1]=0$. The implementation is shown in Figure 2,

Under this implementation, if the register is initialized with the value 1 , the adversary's best strategy is to schedule as follows: First $p$ reads "up" seeing $A[0]=0$ and then $A[1]=1$. Next, $w$ takes all of its steps, then finally $p$ takes its remaining steps where it reads "down". With probability $1 / 2, w$ executed $A[0]$.write $(1)$ and $p$ will return 0 ; with probability $1 / 2$, $w$ executed $A[2]$.write(1) and $p$ will return 1 . Hence the expected value returned by $p$ 's READ is $1 / 2$.

In this example, the adversary makes all its scheduling decisions in advance; it does not exploit knowledge of the outcome of coin flips while the computation proceeds. Even reducing the power of the adversary from strong to this weakest oblivious one does not curtail its power sufficiently to retain the expected behaviour of the algorithm when $R$ is an atomic register. 
These examples motivate our central question: What is required to preserve the behaviour of a randomized algorithm when atomic operations are replaced by method calls? The rest of this paper addresses this question.

\section{Model and Definitions}

We consider a distributed shared memory system consisting of a set $\mathcal{P}$ of $n$ processes communicating via a set of globally shared base objects.

A shared object is an instance of a type, which supports some set of operations. Each such operation op consists of an invocation including operation arguments, denoted inv(op), and a matching response including the return value, denoted $r s p(o p)$. A type is defined by a sequential specification, which determines the set of sequences of operations that can occur on any object of that type [HW90. A sequence is valid for object $O$ if it is in the sequential specification of the type of $O$.

In this paper, we restrict ourselves to deterministic types (except for coin objects as described below). I.e., if $o p_{1}, \ldots, o p_{k}$ and $o p_{1}, \ldots, o p_{k-1}, o p_{k}^{\prime}$ are valid sequences and $i n v\left(o p_{k}\right)=i n v\left(o p_{k}^{\prime}\right)$, then $\operatorname{rsp}\left(o p_{k}\right)=\operatorname{rsp}\left(o p_{k}^{\prime}\right)$.

A process is a sequential thread of control that invokes operations on shared base objects and receives the responses of such operations. Processes also have access to independent random experiments. Let $\Omega$ be an arbitrary countable set, called the coin flip domain. A process step can invoke a flip operation (with no arguments) on a coin object, which returns a coin flip in $\Omega$ as the matching response.

An implementation of a target type $T$ is a distributed method using other implemented or base objects. It takes as input the description of an operation invocation, and outputs a response, such that if multiple processes call the method multiple times sequentially, then the resulting sequence of method invocations and responses matches the sequential specification of $T$. An implementation is deterministic, if it uses no coin objects; in this paper we consider only deterministic implementations of types. An implemented object is a method that implements a type.

Each individual process $p$ executes its program by executing a sequence of operations on shared objects, where the first operation is fixed and the $k$-th operation invocation, $k>1$, is a function of the responses $p$ received from the preceding $k-1$ operations (including flip operations).

Steps of multiple processes interleave, resulting in a history $H$, which is a sequence of steps, i.e., invocations and responses corresponding to the operations executed by all processes on all base objects and all implemented objects.

Thus, the projection of $H$ onto the steps of any process, $p$, denoted $H \mid p$, is a sequence of steps consistent with $p$ 's program.

We say that an operation $o p$ is atomic in history $H$, if op's invocation is either the last step in $H$, or else is followed immediately in $H$ by a matching response. (Note that in related literature, an atomic operation is typically represented by a single event. However, for technical reasons that become more clear in Section [3, the invocation/response representation is more convenient in this paper.) Operations on implemented objects are never atomic, while operations on base objects may or may not be atomic. (We assume that an operation on an implemented object internally applies at least one base object operation.) A history $H$ is sequential if all operations in $H$ are atomic.

A history, $H$, defines a partial happens before order $\prec_{H}$ on its operations, where, for operations $o p$ and $o p^{\prime}, o p \prec_{H} o p^{\prime}$ if and only if in $H$ the response of $o p$ occurs before the invocation of $o p^{\prime}$. 
(The relation $\prec_{H}$ is a total order if and only if $H$ is sequential.)

A sequential history, $H$, is valid if, for any object $O$, the projection of $H$ onto the steps associated with $O$, denoted $H \mid O$, is in the sequential specification of the type of $O$. The new history formed from concatenating history $H$ to the end of history $G$ is denoted $G \circ H$.

A history that arises from an algorithm that uses an implemented object $O$ can be interpreted as a history $\Gamma(H)$ of the same algorithm using a base object of the same type: $\Gamma(H)$ is obtained from $H$ by omitting, for each operation $o p$ on $O$, say by process $p$, all the steps that appear in $H \mid p$ after the invocation $i n v(o p)$ and before the matching response $r \operatorname{sp}(o p)$. Thus, for each operation $o p$ on $O$ in $\Gamma(H)$, inv $(o p)$ corresponds to the method invocation that simulates operation $o p$, $r s p(o p)$ corresponds to the response of that method call, and all operations on the base objects within the method call are omitted. The set of histories of an implementation is the set of histories where processes access an object instantiated using the implementation (and no other implemented object).If $\mathcal{H}$ is a set of histories, then $\Gamma(\mathcal{H})=\{\Gamma(H) \mid H \in \mathcal{H}\}$ denotes the set of interpretations of histories in $\mathcal{H}$.

For correctness, an interpreted history should "correspond" to one that could arise from an atomic object. This is captured by the correctness property called linearizability [HW90. (Note that in literature sometimes the term atomic object is used to denote a linearizable object, see e.g. [Lyn96].) An operation, op, is complete in a history $H$ if $H$ contains both inv(op) and a matching $r s p(o p)$. Since a process is a sequential thread of control, we see that every operation in $H \mid p$, except possibly the last one, is complete. A linearization of a history $H$ is a valid sequential history $H^{\prime}$ that contains all completed operations of $H$ and possibly some non-completed ones (with matching responses added), and where $\prec_{H^{\prime}}$ extends $\prec_{H}$. A history $H$ is linearizable if it has at least one linearization. (Note that a history containing operations on implemented objects is not linearizable in general because it encodes operations on base objects, but its interpretation might be linearizable.)

An implementation of a shared object type is linearizable if its set of histories contains only histories whose interpretations are linearizable.

Flip operations on a coin object are always atomic, and return a value from the set $\Omega$ defined earlier. A vector $\vec{c}=\left(c_{1}, c_{2}, \ldots\right) \in \Omega^{\infty}$ is called a coin flip vector. History $H$ observes the coin flip vector $\vec{c}=\left(c_{1}, c_{2}, \ldots\right)$, if the $i$-th flip operation in $H$ returns value $c_{i}$. For a history $H$ that contains $k$ flip operations, let $H[k]$ denote the prefix of $H$ that ends with the $k$-th invocation of a flip operation; if fewer than $k$ flips occur during $H$, then $H[k]$ denotes $H$.

The order in which steps of processes interleave is given by a schedule, which is a (possibly infinite) sequence of process IDs. History $H$ observes schedule $\sigma=\left(\sigma_{1}, \sigma_{2}, \ldots\right)$, if in $H$ the $i$-th step is one executed by process $\sigma_{i}$.

Schedules are generated by an adversary. Typically, adversaries take the past execution into account to schedule the next process. We are concerned primarily with two adversaries. Informally, a weak adversary cannot intervene between a flip operation and the next operation invocation by the same process. This means that in any history, any flip operation by a process $p$ is immediately followed by an invocation step by $p$. In contrast, a strong adversary can use the response of the coin flip to determine which process takes the next step. The following definitions serve to unify these adversaries, and can easily be seen to capture these informal notions. An adversary is a mapping $\mathcal{A}: \Omega^{\infty} \rightarrow \mathcal{P}^{\infty}$. An algorithm $\mathcal{M}$ together with an adversary $\mathcal{A}$ and a coin flip vector $\vec{c}=\left(c_{1}, c_{2}, \ldots\right) \in \Omega^{\infty}$ generates the unique history, denoted $H_{\mathcal{M}, \mathcal{A}, \vec{c}}$, that observes the schedule $\mathcal{A}(\vec{c})$ and the coin flip vector $\vec{c}$, and where all processes perform steps as dictated by $\mathcal{M}$. 
- An adversary without additional restrictions is called an offline adversary. (An offline adversary can "see" all the coin flips in advance and can use them to make current scheduling decisions.)

- Adversary $\mathcal{A}$ is strong for algorithm $\mathcal{M}$ if, for any two coin flip vectors $\vec{c}$ and $\vec{d}$ that have a common prefix of length $k, H_{\mathcal{M}, \mathcal{A}, \vec{c}}[k+1]=H_{\mathcal{M}, \mathcal{A}, \vec{d}}[k+1]$. (A strong adversary cannot use future coin flips to make current scheduling decisions.)

- Adversary $\mathcal{A}$ is weak for algorithm $\mathcal{M}$ if it is strong for algorithm $\mathcal{M}$ and is additionally constrained so that, in $H_{\mathcal{M}, \mathcal{A}, \vec{c}}$, every flip by process $p$ is followed immediately by the invocation of some operation by $p$. (A weak adversary cannot use future coin flips or the current coin flip to make the next scheduling decision.)

- Adversary $\mathcal{A}$ is oblivious if $\mathcal{A}$ is a constant function, that is, $\mathcal{A}(\vec{c})$ is the same for all $\vec{c} \in \Omega^{\infty}$. (An oblivious adversary cannot use coin flips at all to make scheduling decisions.)

A strong adversary is commonly considered in the distributed algorithm literature. Our weak adversary is similar to other adversaries in the literature, such as that assumed by Chor, Israeli and Li [CIL87, and further discussed by Abrahamson [Abr88. However, while their adversary cannot intervene between flip operations and writes, it can intervene between flips and reads. (No other atomic operations are considered.) Our goal is to compare the behaviour of systems with atomic objects to those with implemented objects, for arbitrary objects that could support stronger operations than just reads and writes. Consequently, we assume that an adversary treats all operations consistently; it cannot intervene between a flip and some operations but not others. Furthermore, always binding a flip operation to the next step of the same process, instead of binding only if that next step is a write, serves to strengthen our impossibility result for weak adversaries in Section 5 .

As we compare the powers of different adversaries in the remainder of the paper, we will refer repeatedly to the following notion of equivalence:

Definition 3.1. Let $\mathcal{M}$ and $\mathcal{M}^{\prime}$ be two algorithms and $\mathcal{A}$ and $\mathcal{A}^{\prime}$ be two adversaries. We say that $(\mathcal{M}, \mathcal{A})$ and $\left(\mathcal{M}^{\prime}, \mathcal{A}^{\prime}\right)$ are equivalent if for any coin flip vector $\vec{c}$, there exists a sequential history that is a linearization of $\Gamma\left(H_{\mathcal{M}, \mathcal{A}, \vec{c}}\right)$ and of $\Gamma\left(H_{\mathcal{M}^{\prime}, \mathcal{A}^{\prime}, \vec{c}}\right)$.

Some of the results discussed in Sections 3 and 5 refer to well-known progress requirements. An implementation of a shared object type is wait-free if in any history, each method call incurs a finite number of steps. An implementation is lock-free if in any history, either each method call takes finitely many steps, or else infinitely many method calls complete. An implementation is terminating if in any history, either each method call takes finitely many steps, or else some process that takes finitely many steps invokes a method call that it does not complete.

In this section, we discuss a novel technique for limiting the additional power a strong adversary may gain against an algorithm when atomic objects used by the algorithm are replaced with implemented objects.

\section{Strong Linearizability}

We define a correctness property stronger than linearizability, called strong linearizability, and prove that under any strong adversary, strongly linearizable implementations of shared objects preserve the probability space of computations of an algorithm using such objects. We also show that strong 
linearizability maintains locality and composability-powerful properties that facilitate algorithm design.

For a set of histories $\mathcal{H}$, let close $(\mathcal{H})$ denote the prefix-closure of $\mathcal{H}$. That is, $G \in \operatorname{close}(\mathcal{H})$ if and only if there is a sequence, $S$, of invocation and response steps such that $G \circ S \in \mathcal{H}$. (Recall that the operator $\circ$ denotes concatenation.) Consider a function $f$ that maps a set $\mathcal{H}$ of histories to a set $\mathcal{H}^{\prime}$ of histories. We say that $f$ is prefix preserving, if for any two histories $G, H \in \mathcal{H}$, where $G$ is a prefix of $H, f(G)$ is a prefix of $f(H)$.

Definition 4.1. A set of histories $\mathcal{H}$ is strongly linearizable if there exists a function $f$ mapping histories in close $(\mathcal{H})$ to sequential histories, such that

$(L)$ for any $H \in \operatorname{close}(\mathcal{H}), f(H)$ is a linearization of the interpreted history $\Gamma(H)$, and

(P) $f$ is prefix-preserving.

A function satisfying properties $(L)$ and $(P)$ is called a strong linearization function for $\mathcal{H}$.

An implementation of a type is strongly linearizable if the set of histories formed by interpreting each history in the set of histories of the implementation is strongly linearizable.

We emphasize some differences between the concept of linearizability and strong linearizability:

1. In order to determine whether an implementation of a type is linearizable it suffices to look at every single history individually; However, property $(\mathrm{P})$ from the definition of strong linearizability is defined for sets of histories, so we have to consider all possible histories together.

2. Linearizability is defined in terms of interpreted histories. I.e., it does not matter how an object is implemented, as long as all possible sequences of high-level invocations and responses satisfy the linearizability property. For strong linearizability the low-level (i.e., noninterpreted) histories have to satisfy property $(\mathrm{P})$, so the implementation of the object seems to be more important.

In the following we consider sets of histories $\mathcal{H}$ that are generated by a (fixed) strong adversary $\mathcal{A}$ for a given algorithm $\mathcal{M}$. It will prove helpful to note that in this case it does not make a difference for the strong linearizability of $\mathcal{H}$ whether $\mathcal{H}$ is a set of low-level histories or of interpreted histories:

Observation 4.2. Let $\mathcal{M}$ be an algorithm and $\mathcal{A}$ a strong adversary. Then $\mathcal{H}:=$ $\left\{H_{\mathcal{M}, \mathcal{A}, \vec{c}} \mid \vec{c} \in \Omega^{\infty}\right\}$ is strongly linearizable if and only if $\Gamma(\mathcal{H})=\left\{\Gamma\left(H_{\mathcal{M}, \mathcal{A}, \vec{c}}\right) \mid \vec{c} \in \Omega^{\infty}\right\}$ is strongly linearizable.

Proof. Assume w.l.o.g. that $\mathcal{H}$ is prefix-closed (and then so is $\Gamma(\mathcal{H})$ ). First note that for any $H, H^{\prime} \in \mathcal{H}$ the following is true:

$$
\text { If } \Gamma(H)=\Gamma\left(H^{\prime}\right) \text {, then either } H \text { is a prefix of } H^{\prime} \text { or vice versa. }
$$

Let $G$ be the longest common prefix of $H$ and of $H^{\prime}$. For the purpose of a contradiction assume that $G$ is a proper prefix of $H$ and of $H^{\prime}$. Since $H$ and $H^{\prime}$ are generated from the same algorithm $\mathcal{M}$ and the scheduling of a strong adversary, $G$ must end with the invocation of a flip operation $f l$. Hence, the response of that flip operation is in $H$ and $H^{\prime}$ but its return value is different in these two histories. But since all object implementations are deterministic, $f l$ must occur (and respond) in $\Gamma(H)=\Gamma\left(H^{\prime}\right)$ - a contradiction. 
Now suppose that $\mathcal{H}$ is strongly linearizable. Let $f$ be a strong linearization function for $\mathcal{H}$. For any history $H^{\prime} \in \Gamma(\mathcal{H})$ let $\max _{\mathcal{H}}\left(H^{\prime}\right)$ be the longest history in $\mathcal{H}$ with $\Gamma\left(\max _{\mathcal{H}}\left(H^{\prime}\right)\right)=H^{\prime} .(\operatorname{By}($ 困) all histories $H$ with $\Gamma(H)=H^{\prime}$ are prefixes of $\max _{\mathcal{H}}\left(H^{\prime}\right)$.) Define $f^{\prime}\left(H^{\prime}\right)=f\left(\max _{\mathcal{H}}\left(H^{\prime}\right)\right)$. Then $f^{\prime}$ is a strong linearization function of $\Gamma(\mathcal{H})$ : If $G^{\prime}$ is a prefix of $H^{\prime}$, for $G^{\prime}, H^{\prime} \in \Gamma(\mathcal{H})$, then $\max _{\mathcal{H}}\left(G^{\prime}\right)$ is a prefix of $\max _{\mathcal{H}}\left(H^{\prime}\right)$ and so $f^{\prime}\left(G^{\prime}\right)=f\left(\max _{\mathcal{H}}\left(G^{\prime}\right)\right)$ is a prefix of $f^{\prime}\left(H^{\prime}\right)=f\left(\max _{\mathcal{H}}\left(H^{\prime}\right)\right)$. Moreover, since $f$ satisfies property $(\mathrm{L}), f^{\prime}\left(H^{\prime}\right)$ is a linearization of $\Gamma\left(\max _{\mathcal{H}}\left(H^{\prime}\right)\right)=H^{\prime}$. Thus, $f^{\prime}$ satisfies properties $(\mathrm{P})$ and $(\mathrm{L})$.

Now suppose that $\Gamma(\mathcal{H})$ is strongly linearizable and that $g$ is a strong linearization function for it. For each history $H \in \mathcal{H}$ we define $g^{\prime}(H):=g(\Gamma(H))$. Then it is immediate that $g^{\prime}$ inherits properties $(\mathrm{P})$ and $(\mathrm{L})$ from $g$, so $g^{\prime}$ is a strong linearization function for $\mathcal{H}$.

\subsection{Strong Linearizability is Necessary}

In the following we show that if the power of the set of (at most) strong adversaries is not enhanced by the implemented objects, then the algorithm using implemented objects generates a strongly linearizable set of histories.

Theorem 4.3. Let $\mathcal{M}$ be an algorithm that uses only atomic objects, and let $\mathcal{M}^{\prime}$ be the algorithm obtained from $\mathcal{M}$ by replacing some objects with linearizable implementations. Further, let $\mathcal{A}^{\prime}$ be an adversary that is strong for $\mathcal{M}^{\prime}$. If there exists an adversary $\mathcal{A}$ that is strong for $\mathcal{M}$ such that $(\mathcal{A}, \mathcal{M})$ and $\left(\mathcal{A}^{\prime}, \mathcal{M}^{\prime}\right)$ are equivalent, then $\mathcal{H}^{\prime}=\left\{H_{\mathcal{M}^{\prime}, \mathcal{A}^{\prime}, \vec{c}} \mid \vec{c} \in \Omega^{\infty}\right\}$ is strongly linearizable.

Due to Observation 4.2, it suffices to consider only interpreted histories when proving this theorem. Thus, in the following proof we only consider interpreted histories. For the ease of notation we simply write $H$ instead of $\Gamma(H)$ for every history $H$ considered.

Let $\mathcal{H}^{*}=\operatorname{close}\left(\mathcal{H}^{\prime}\right)$. Since $(\mathcal{A}, \mathcal{M})$ and $\left(\mathcal{A}^{\prime}, \mathcal{M}^{\prime}\right)$ are equivalent, and all histories of $\mathcal{M}$ are sequential, each history $H^{\prime}=H_{\mathcal{M}^{\prime}, \mathcal{A}^{\prime}, \vec{c}}$, where $\vec{c} \in \Omega^{\infty}$, has a linearization $\ell\left(H^{\prime}\right)=H_{\mathcal{M}, \mathcal{A}, \vec{c}}$. Let $H^{\prime} \in \mathcal{H}^{\prime}$ and let $G^{\prime} \in \mathcal{H}^{*}$ be a prefix of $H^{\prime}$. Define $g\left(G^{\prime}, H^{\prime}\right)$ to be the shortest prefix of $\ell\left(H^{\prime}\right)$ that contains all operations that complete in $G^{\prime}$. Two claims help clarify the proof of Theorem 4.3 ,

Claim 4.4. $G=g\left(G^{\prime}, H^{\prime}\right)$ is a linearization of $G^{\prime}$.

Proof. Suppose that $o p \prec_{G^{\prime}} o p^{\prime}$. Since $G^{\prime}$ is a prefix of $H^{\prime}$, op $\prec_{H^{\prime}} o p^{\prime}$ holds, and thus $o p^{\prime} \nprec_{H} o p$ for the linearization $H=\ell\left(H^{\prime}\right)$ of $H^{\prime}$. History $G$ is a prefix of $H$, and so $o p^{\prime} \nprec_{G} o p$.

By construction, $G$ contains all completed operations from $G^{\prime}$, and so it suffices to show that if $G$ contains an operation, then that operation's invocation occurs also in $G^{\prime}$. For contradiction let $o p$ be any operation in $G$ such that $i n v(o p)$ does not occur in $G^{\prime}$. By construction, some operation $o p^{\prime}$ must follow $o p$ in $G$, and so $o p^{\prime}$ completes in $G^{\prime}$ (otherwise, $G$ would not be the shortest prefix of $H$ that contains all operations that complete in $G^{\prime}$ ). However, since $o p \prec_{G} o p^{\prime}$ and $G$ is a prefix of $H$, we know that $o p \prec_{H} o p^{\prime}$ and thus $o p^{\prime} \nprec_{H^{\prime}} o p$. Hence, in $H^{\prime} r s p\left(o p^{\prime}\right)$ occurs only after $i n v(o p)$. But then, since $G^{\prime}$ is a prefix of $H^{\prime}$ that contains $r s p\left(o p^{\prime}\right)$, inv(op) must occur in $G^{\prime}$ as well-a contradiction.

Claim 4.5. Let $H_{c}^{\prime}=H_{\mathcal{A}^{\prime}, \mathcal{M}^{\prime}, \vec{c}}, H_{d}^{\prime}=H_{\mathcal{A}^{\prime}, \mathcal{M}^{\prime}, \vec{d}} \in \mathcal{H}^{\prime}$ and $G^{\prime} \in \mathcal{H}^{*}$ be a common prefix of both $H_{c}^{\prime}$ and $H_{d}^{\prime}$. Then $g\left(G^{\prime}, H_{c}^{\prime}\right)=g\left(G^{\prime}, H_{d}^{\prime}\right)$.

Proof. The claim is trivially true if $H_{c}^{\prime}=H_{d}^{\prime}$, so assume that $H_{c}^{\prime} \neq H_{d}^{\prime}$. Suppose that the longest common prefix of $\vec{c}$ and $\vec{d}$ has length $k$. Since $G^{\prime}$ is a common prefix of $H_{c}^{\prime}$ and $H_{d}^{\prime}$, it cannot contain 
the response of the $(k+1)$-th flip operation. Thus, $G^{\prime}$ is a prefix of both $H_{c}^{\prime}[k+1]$ and $H_{d}^{\prime}[k+1]$, and furthermore $H_{c}^{\prime}[k+1]=H_{d}^{\prime}[k+1]$ since $\mathcal{A}^{\prime}$ is a strong adversary. Let $G_{c}=g\left(G^{\prime}, H_{c}^{\prime}\right)$ and $G_{d}=g\left(G^{\prime}, H_{d}^{\prime}\right)$. We will show that neither $G_{c}$ nor $G_{d}$ contains the $(k+1)$-th coin flip. Suppose for contradiction that $G_{c}$ does. (The proof for $G_{d}$ is analogous.) Since the coin flip is not complete in $G^{\prime}$, by construction of $G_{c}$ some operation op that is complete in $G^{\prime}$ must follow the coin flip in $G_{c}$. Since $G_{c}$ is a linearization of $G^{\prime}$ by Claim 4.4, the invocation of the coin flip must precede the response of $o p$ in $G^{\prime}$. But that contradicts $G^{\prime}$ being a prefix of $H_{c}^{\prime}[k+1]$, which ends with the coin flip's invocation. Now since $G_{c}$ does not contain the $(k+1)$-st coin flip, it is a prefix not only of $\ell\left(H_{c}^{\prime}\right)$ but also of $\ell\left(H_{c}^{\prime}[k+1]\right)$, and similarly $G_{d}$ is a prefix of $\ell\left(H_{d}^{\prime}[k+1]\right)$. Since $H_{c}^{\prime}[k+1]=H_{d}^{\prime}[k+1]$ holds, as noted earlier, this implies that $G_{c}=G_{d}$.

Proof of Theorem 4.3. For all histories $G^{\prime} \in \mathcal{H}^{*}$, we define $f\left(G^{\prime}\right)=g\left(G^{\prime}, H^{\prime}\right)$, where $H^{\prime}$ is an arbitrary history in $\mathcal{H}^{\prime}$ such that $G^{\prime}$ is a prefix of $H^{\prime}$. (By Claim 4.5, all such histories $H^{\prime}$ yield the same $g\left(G^{\prime}, H^{\prime}\right)$.) We show that $f$ is a linearization function for $\mathcal{H}^{\prime}$. By Claim 4.4, $f$ satisfies property (L), so it suffices to show that it also satisfies property (P). Let $F^{\prime}, G^{\prime} \in \mathcal{H}^{*}$, such that $F^{\prime}$ is a prefix of $G^{\prime}$. Choose an arbitrary history $H^{\prime} \in \mathcal{H}^{\prime}$ such that $G^{\prime}$ is a prefix of $H^{\prime}$. By construction and Claim 4.5, $f\left(G^{\prime}\right)$ and $f\left(F^{\prime}\right)$ are the shortest prefixes of $\ell\left(H^{\prime}\right)$ that contain all completed operations in $G^{\prime}$ and $F^{\prime}$, respectively. Since the set of completed operations in $F^{\prime}$ is a subset of the completed operations in $G^{\prime}, f\left(F^{\prime}\right)$ is a prefix of $f\left(G^{\prime}\right)$, completing the proof of Theorem 4.3 ,

\subsection{Strong Linearizability is Sufficient for the Strong Adversary}

Under strong linearizability the strong adversary is prevented from using the outcome of the flip to schedule future events in such a way that they influence the order of past operations in a linearization, because, once a coin is flipped, the operations that precede the coin flip in the linearization are already determined. This is made precise in the following theorem, the proof of which appears later in this subsection.

Theorem 4.6. Let $\mathcal{M}$ be an algorithm that uses only atomic objects, and let $\mathcal{M}^{\prime}$ be the algorithm obtained from $\mathcal{M}$ by replacing some atomic objects with strongly linearizable implementations. For any adversary $\mathcal{A}^{\prime}$ that is strong for $\mathcal{M}^{\prime}$, there exists an adversary $\mathcal{A}$ that is strong for $\mathcal{M}$, such that $(\mathcal{M}, \mathcal{A})$ and $\left(\mathcal{M}^{\prime}, \mathcal{A}^{\prime}\right)$ are equivalent.

The proof is postponed to a later section.

\subsection{Normalized Strong Linearizations.}

Let $\mathcal{H}_{\mathcal{M}, \mathcal{A}}$ denote the set of all interpreted histories that are generated by an algorithm $\mathcal{M}$ and the adversary $\mathcal{A}$ over all coin flip vectors. That is $\mathcal{H}_{\mathcal{M}, \mathcal{A}}=\left\{\Gamma\left(H_{\mathcal{M}, \mathcal{A}, \vec{c}}\right) \mid \vec{c} \in \Omega^{\infty}\right\}$. A natural way to try to prove Theorem 4.6 would be to apply the strong linearization function to each history in the set $\mathcal{H}_{\mathcal{M}^{\prime}, \mathcal{A}^{\prime}}$ to obtain a set $\mathcal{H}$ of linearizations of $\mathcal{H}_{\mathcal{M}^{\prime}, \mathcal{A}^{\prime}}$. Then it would suffice to prove that there is a strong adversary $\mathcal{A}$ that can generate the histories in $\mathcal{H}$.

Unfortunately, this is not always possible. For example, consider an algorithm, $\mathcal{M}$, where process $p$ (respectively, $q$ ) executes the single operation $o p_{p}$ (resp. $o p_{q}$ ) and process $r$ first executes $o p_{r}$ and then executes a flip operation, $c f$. Suppose a strong adversary schedules an implementation 
of $\mathcal{M}$ so that, for coin flip $i \in\{0,1\}$, it produces the history:

$$
H_{i}^{\prime}=i n v\left(o p_{p}\right), i n v\left(o p_{q}\right), i n v\left(o p_{r}\right), r s p\left(o p_{r}\right), i n v\left(c f_{r}\right), r s p\left(c f_{r}, i\right), r s p\left(o p_{p}\right), r s p\left(o p_{q}\right)
$$

Let $G^{\prime}$ be the common prefix of $H_{0}^{\prime}$ and $H_{1}^{\prime}$ that ends with $i n v\left(c f_{r}\right)$. Define the function $f$ on $\left\{G^{\prime}, H_{0}^{\prime}, H_{1}^{\prime}\right\}$ by:

$$
\begin{aligned}
f\left(G^{\prime}\right) & =i n v\left(o p_{r}\right), r s p\left(o p_{r}\right) \\
f\left(H_{0}^{\prime}\right) & =i n v\left(o p_{r}\right), r s p\left(o p_{r}\right), i n v\left(o p_{p}\right), r s p\left(o p_{p}\right), i n v\left(o p_{q}\right), r s p\left(o p_{q}\right), i n v\left(c f_{r}\right), r s p\left(c f_{r}, 0\right) \\
f\left(H_{1}^{\prime}\right) & =i n v\left(o p_{r}\right), r s p\left(o p_{r}\right), i n v\left(c f_{r}\right), r s p\left(c f_{r}, 1\right), i n v\left(o p_{q}\right), r s p\left(o p_{q}\right), i n v\left(o p_{p}\right), r s p\left(o p_{p}\right)
\end{aligned}
$$

Then, according to Definition 4.1, $f$ can be extended to a strong linearization function for $\left\{H_{0}^{\prime}, H_{1}^{\prime}\right\}$, but the histories $f\left(H_{0}^{\prime}\right)$ and $f\left(H_{1}^{\prime}\right)$ cannot both be produced by the same strong adversary. This difficulty is remedied by proving that whenever such a problematic strong linearization function for a set of histories $\mathcal{H}$ occurs, there is another strong linearization for $\mathcal{H}$ that avoids this problem. The idea is to move coin flips in $f(H)$ to the earliest point possible, without violating the happens-before order of $H$. The following technical lemma makes this precise.

Definition 4.7. Let $\mathcal{H}$ be a strongly linearizable set of histories. A normalized strong linearization function for $\mathcal{H}$ is any strong linearization function $f^{*}$ for $\mathcal{H}$ such that:

$(N)$ for any history $H \in$ close $(\mathcal{H})$, if in $f^{*}(H)$ some flip operation cf immediately follows some other operation op, then op $\prec_{H} c f$.

Lemma 4.8. For any strongly linearizable set $\mathcal{H}$ of histories there exists a normalized strong linearization function.

Proof. Let $f$ be a strong linearization function for $\mathcal{H}$. Let $H \in \operatorname{close}(\mathcal{H})$ be an arbitrary history and let $c f_{1}, c f_{2}, \ldots$ be the flip operations in $H$, occurring in that order. We obtain $f^{*}(H)$ from $f(H)$ as follows:

1. Let $H^{\prime}=f(H)$. As long as the response of the last operation op in $H^{\prime}$ does not appear in $H$, remove op from $H^{\prime}$.

2. Remove all flip operations from $H^{\prime}$.

3. For $i=1,2, \ldots$, insert $c f_{i}$ at the earliest possible position in $H^{\prime}$, where it doesn't violate $\prec_{H}$.

Let $f^{\prime}(H)$ be the history obtained from $f(H)$ after execution of Step 1. It is immediate from Step 1 that $f^{\prime}(H)$ satisfies the following:

The response of the last operation in $f^{\prime}(H)$ occurs in $H$.

Moreover, $f^{\prime}$ satisfies property $(\mathrm{L})$ : If $f(H)$ is a linearization of $\Gamma(H)$ that ends with an operation $o p$ which does not complete in $H$, then we can remove $o p$ without destroying validity or changing the order of any of the remaining pairs of operations in $f(H)$. Similarly, property $(\mathrm{P})$ is maintained: Since removals from histories occur only at the end, such removals can only violate property $(\mathrm{P})$ for a history $H$ with prefix $G$, if an operation op is removed from $f(H)$ and op occurs also in $f(G)$ but is not removed from $f(G)$. If that were to happen, then $f(G)=f(H)$ since $f(G)$ is a prefix of $f(H)$ and $o p$ is the last operation of both histories. But if op is removed from the end of $f(H)$, 
then op does not complete during $H$. And then op does not complete in $G$, either, and so it is removed from $f(G)$, too.

Now suppose that $f^{\prime}$ satisfies $(\mathrm{L}),(\mathrm{P})$, and (因) after Step 1. We show that after Steps 2 and 3 , the resulting mapping, which we call $f^{*}$, satisfies $(\mathrm{L}),(\mathrm{P})$, and $(\mathrm{N})$.

First we show that $f^{*}$ satisfies $(\mathrm{N})$ : Suppose in Step 3, the flip operation $c f_{i}$ is inserted immediately after some other operation $o p$. Then $o p \prec_{H} c f_{i}$, or else $c f_{i}$ could have been inserted before $o p$. For all flip operations $c f_{j}$ that are inserted after $c f_{i}, c f_{i} \prec_{H} c f_{j}$ holds, and so they are inserted into $H^{\prime}$ behind $c f_{i}$. Thus, at the end of Step 3, $c f_{i}$ immediately follows $o p$.

Next we show that $f^{*}$ satisfies $(\mathrm{L})$, i.e., that $f^{*}(H)$ is a linearization of $\Gamma(H)$. Since $f^{\prime}(H)$ and $f(H)$ differ only in the position of the (atomic) flips, and the relative order of flips is preserved, $f(H)$ is valid. Now consider two operations $o p, o p^{\prime}$ that both appear in $f^{*}(H)$, and where $o p \prec_{H} o p^{\prime}$. Then $o p$ and $o p^{\prime}$ both occur in $f^{\prime}(H)$, and since $f^{\prime}$ satisfies (L), op $\prec_{f^{\prime}(H)} o p^{\prime}$. If neither of the two operations is a flip, then the order of them is preserved when we construct $f^{*}(H)$. If one of the operations is a flip, then the insertion rule guarantees that the flip operation is inserted in such a way that it does not violate $\prec_{H}$. Finally, only operations that don't complete in $H$ are removed from $f(H)$ in Step 1, so $f^{\prime}(H)$ and thus also $f^{*}(H)$ contain all operations that complete in $H$.

It remains to show that $f^{*}$ satisfies $(\mathrm{P})$. Let $G$ be an arbitrary prefix of $H$ and let $O$ be the set of non-flip operations in $H$. Further, let $O_{i}=O \cup\left\{c f_{1}, \ldots, c f_{i}\right\}$, for $0 \leq i \leq z$, where $z$ is the number of flip operations in $H$. We show by induction on $i, 0 \leq i \leq z$, that

$$
f^{*}(G) \mid O_{i} \text { is a prefix of } f^{*}(H) \mid O_{i} .
$$

First consider the base case, $i=0$. Note that $O_{0}=O$. By construction, $f^{*}(G)$ contains exactly the same set of operations as $f^{\prime}(G)$, and $f^{*}(H)$ contains the same set of operations as $f^{\prime}(H)$. Since the order of non-flip operations does not change during Steps 2 and $3, f^{*}(G)\left|O=f^{\prime}(G)\right| O$, and $f^{*}(H)\left|O=f^{\prime}(H)\right| O$. Since $f^{\prime}$ satisfies $(\mathrm{P})$, it follows that $f^{*}(G) \mid O$ is a prefix of $f^{*}(H) \mid O$.

Now suppose $i \geq 1$ and that $f^{*}(G) \mid O_{i-1}$ is a prefix of $f^{*}(H) \mid O_{i-1}$. Let $G^{*}:=f^{*}(G) \mid O_{i}$ and $H^{*}=f^{*}(H) \mid O_{i}$. Below, we prove the following statement:

$$
\text { for any operation op in } G^{*}: c f_{i} \prec_{H^{*}} \text { op } \Leftrightarrow c f_{i} \prec_{G^{*}} o p \text {. }
$$

Thus, if $c f_{i}$ appears in both $G^{*}$ and $H^{*}$, it appears in the same position. On the other hand, if $c f_{i}$ does not appear in $G^{*}$, then $c f_{i} \nprec_{G^{*}} o p$ for all operation op that appear in $G^{*}$; hence, in $H^{*} c f_{i}$ is not inserted before some operation op that appears in $G^{*}$. Therefore, from (1) we can conclude that $G^{*}$ is a prefix of $H^{*}$, and so it suffices to prove (1).

First suppose $c f_{i} \prec_{G^{*}} o p$, and for the purpose of a contradiction assume $c f_{i} \nprec_{H^{*}} o p$. Since all operations in $G^{*}$ occur also in $H^{*}, o p \prec H^{*} c f_{i}$. Let $o p^{\prime}$ be the operation that immediately precedes $c f_{i}$ in $H^{*}$. Then $o p \preceq H^{*} o p^{\prime} 2$ By the semantics of Step 3,op $\prec_{H} c f_{i}$, or else $c f_{i}$ would have been inserted in front of $o p^{\prime}$. Since $c f_{i}$ completes during $G$, and $G$ is a prefix of $H, o p^{\prime}$ completes during $G$ as well. Hence, $o p^{\prime} \prec_{G} c f_{i}$ and since $G^{*}$ is a linearization of $G \mid O_{i}, o p^{\prime} \prec_{G^{*}} c f_{i}$. By induction hypothesis, from $o p \preceq_{H^{*}} o p^{\prime}$, we have $o p \preceq_{G^{*}} o p^{\prime}$ and thus by transitivity $o p \prec_{G^{*}} c f_{i}$-a contradiction.

Now suppose $c f_{i} \prec_{H^{*}} o p$. For the purpose of a contradiction assume that $c f_{i} \nprec_{G^{*}} o p$. Then either $c f_{i}$ does not occur in $G^{*}$ at all or $o p \prec G^{*} c f_{i}$. First consider the latter case. Let $o p^{\prime}$ be the last operation in $G^{*}$ that precedes $c f_{i}$, so $o p \preceq G^{*} o p^{\prime}$. Since $c f_{i}$ was not inserted in front of $o p^{\prime}$, by

\footnotetext{
${ }^{2}$ We write $a \preceq b$ to denote "either $a \prec b$ or $a=b$ ".
} 
the semantics of Step 3 we have $o p^{\prime} \prec_{H} c f_{i}$. Since $H^{*}$ is a linearization of $H \mid O_{i}, o p^{\prime} \prec_{H^{*}} c f_{i}$. By the induction hypothesis, $o p \preceq H^{*} o p^{\prime}$, and so by transitivity $o p \prec H^{*} c f_{i}$-a contradiction.

Finally, assume that $c f_{i}$ is not in $G^{*}$. Let $o p^{\prime}$ be the last operation in $G^{*}$. If $o p^{\prime}$ is a flip, then it appears atomic in $G$, and thus completes in $G$. Otherwise, $o p^{\prime}$ is also the last operation in $f^{\prime}(G)$, and thus by (园), $o p^{\prime}$ completes in $G$. On the other hand, since $c f_{i}$ is a atomic but does not appear in $G^{*}$, it does not appear in $G$, either. Since $G$ is a prefix of $H$, it follows that $o p^{\prime} \prec_{H} c f_{i}$. But then, since $H^{*}$ is a linearization of $H \mid O_{i}, o p^{\prime} \prec_{H^{*}} c f_{i}$. Since $o p$ is in $G^{*}$, but $o p^{\prime}$ is the last operation in $G^{*}$,op $\preceq_{G^{*}} o p^{\prime}$. By the induction hypothesis, $G^{*} \mid\left\{o p, o p^{\prime}\right\}$ is a prefix of $H^{*} \mid\left\{o p, o p^{\prime}\right\}$, and so $o p \preceq H^{*} o p^{\prime} \prec_{H^{*}} c f_{i}$ - a contradiction.

\subsection{Strong Linearizability is a Local Property.}

We could now proceed with the proof of Theorem 4.6. However, the proof is simplified by exploiting a locality property for strong linearizability. Herlihy and Wing proved the following locality property for linearizability [HW90]: A history $H$ over multiple shared objects is linearizable if, for each such object $O$ the history $H \mid O$ is linearizable. Thus, to establish that any result that can arise from an algorithm using implemented objects, is a result that could be produced by the same algorithm using atomic objects, it suffices to show separately for each shared object, $O$, that the implementation of any algorithm over $O$ is linearizable. The analogous property for strong linearizability is given in the following lemma. For a shared implemented object $O$ and a history $H$, we denote by $H \| O$ the history $H$ projected to all steps that any process executes while performing an operation on $O$, i.e., all steps executed by the process during an interval that starts with an invocation on $O$ and ends with the matching response on $O$.

Theorem 4.9. A set of histories $\mathcal{H}$ over implemented objects $O_{1}, O_{2}, \ldots, O_{n}$ is strongly linearizable if for each object $O_{i}, 1 \leq i \leq n$, the set $\mathcal{H}_{i}=\left\{\left(H \| O_{i}\right) \mid H \in \mathcal{H}\right\}$ is strongly linearizable.

Remark 4.10. Typically one would apply the theorem for objects $O_{1}, \ldots, O_{n}$ that are implemented from distinct sets $\mathcal{B}_{1}, \ldots, \mathcal{B}_{n}$ of base objects. This is not required for the correctness of the lemma, though. However, suppose that $n=2$ and the implementations of $O_{1}$ and $O_{2}$ share the same base object $B$. It is easy to construct a history $H$ such that $\left(H \| O_{1}\right) \mid B$ and $\left(H \| O_{2}\right) \mid B$ are valid, but $H \mid B$ is not. Or, it might be the case that $H \mid B$ is valid, but $\left(H \| O_{1}\right) \mid B$ is not. However, even if $\mathcal{H}$ contains such a history, the lemma is correct, because a strong linearization function $f$ maps $H$ to a history $f(H)$ that does not contain any steps on $B$.

Proof of Theorem 4.9. Let $H^{(k)}$ denote the prefix of history $H$ that has length $\min \{k,|H|\}$, and let $\mathcal{H}^{(k)}$ be the subset of all histories in $\operatorname{close}(\mathcal{H})$ that have length at most $k$. First, we use the strong linearization functions $f_{i}$ for each object $O_{i}$ to inductively construct functions $f^{(k)}$, each of which maps each history $H \in \operatorname{close}(\mathcal{H})$ to a sequential history, and satisfies:

(a) for any object $O_{i}, f^{(k)}(H) \mid O_{i}=f_{i}\left(H^{(k)} \| O_{i}\right)$; and

(b) $f^{(k)}$ is a strong linearization function for $\mathcal{H}^{(k)}$; and

(c) $f^{(k)}(H)=f^{(k)}\left(H^{(k)}\right)$; and

(d) if $k \geq 1$, then $f^{(k-1)}(H)$ is a prefix of $f^{(k)}(H)$. 
For the basis, define $f^{(0)}(H)=\varepsilon$, where $\varepsilon$ denotes the empty history. Properties (a) through (d) clearly hold for $f^{(0)}$. Now consider $k \geq 1$ and suppose that $f^{(k-1)}$ satisfies (a)-(d). Construct $f^{(k)}$ as follows. For any history $H$, if $|H|<k$ then define $f^{(k)}(H)=f^{(k-1)}(H)$. Otherwise let $\beta$ be the $k$ 'th step of $H$, and suppose that $\beta$ is a step in $H \| O_{j}$. Since $f_{j}$ is a strong linearization function for $\mathcal{H}_{j}, f_{j}\left(H^{(k-1)} \| O_{j}\right)$ is a prefix of $f_{j}\left(H^{(k)} \| O_{j}\right)$. Consequently, there is a sub-history $\lambda$ (possibly empty) satisfying

$$
f_{j}\left(H^{(k)} \| O_{j}\right)=f_{j}\left(H^{(k-1)} \| O_{j}\right) \circ \lambda
$$

For this case (i.e., $|H| \geq k$ ) define

$$
f^{(k)}(H)=f^{(k-1)}(H) \circ \lambda
$$

For all $k \in \mathbb{N} \cup\{0\}$, property (c) is satisfied because $f^{k}(H)$ is uniquely determined by the prefix of length $\min \{k,|H|\}$ of $H$. Property (d) follows immediately from (3). We now show that properties (a) and (b) are preserved by the inductive step.

Property (a): First consider an object $O_{i}$, where $i \neq j$. Since $\beta$ and all events in $\lambda$ belong to operations on $O_{j}$, we get:

$$
\begin{aligned}
f^{(k)}(H) \mid O_{i} & \stackrel{(\underline{3}}{=}\left(f^{(k-1)}(H) \circ \lambda\right)\left|O_{i}=f^{(k-1)}(H)\right| O_{i} \stackrel{I . H .}{=} f_{i}\left(H^{(k-1)} \| O_{i}\right) \\
& =f_{i}\left(\left(H^{(k-1)} \circ \beta\right) \| O_{i}\right)=f_{i}\left(H^{(k)} \mid O_{i}\right) .
\end{aligned}
$$

Now consider the object $O_{j}$. By definition of $\lambda$, the induction hypothesis, and construction of $f^{(k)}$ :

$$
f_{j}\left(H^{(k)} \| O_{j}\right) \stackrel{(2)}{=} f_{j}\left(H^{(k-1)} \| O_{j}\right) \circ \lambda \stackrel{I . H}{=}\left(f^{(k-1)}(H) \mid O_{j}\right) \circ \lambda=\left(f^{(k-1)}(H) \circ \lambda\right)\left|O_{j}=f^{(k)}(H)\right| O_{j} .
$$

Property (b): We first show that the projection of $f^{(k)}$ on $\mathcal{H}^{(k)}$ satisfies property $(\mathrm{P})$. Let $H$ be an arbitrary history of length at most $k$, and let $G$ be a prefix of $H$. If $|G|=k$, then $G=H$ so trivially $f^{(k)}(G)$ is a prefix of $f^{(k)}(H)$. Now consider the case $|G|<k$, and thus $G=G^{(k-1)}$. Then by construction, $f^{(k-1)}(G)=f^{(k)}(G)$, and so

$$
f^{(k)}(G)=f^{(k)}\left(G^{(k-1)}\right)=f^{(k)}(G)=f^{(k-1)}\left(G^{(k-1)}\right),
$$

which by the induction hypothesis is a prefix of

$$
f^{(k-1)}\left(H^{(k-1)}\right) \stackrel{(c)}{=} f^{(k-1)}(H)
$$

By (d), this is a prefix of $f^{(k)}(H)$.

We now prove that $f^{(k)}$ satisfies property (L) for every history $H \in \mathcal{H}^{(k)}$. Consider an operation $o p$ on some object $O_{i}$ that completes during $H$ and thus also during $\Gamma(H)$. Then op completes during $\Gamma\left(H \| O_{i}\right)$, and also during its linearization $f_{i}\left(H \| O_{i}\right)$. Thus, by (a) op completes during $f^{(k)}(H)$. Now consider two operations $o p_{1}, o p_{2}$ in $\Gamma(H)$ such that $o p_{1} \prec_{H} o p_{2}$. For the purpose of a contradiction, assume that $o p_{2}$ appears before $o p_{1}$ in $f^{(k)}(H)$. First consider the case that 
$o p_{1}$ and $o p_{2}$ both appear in $f^{(k-1)}(H)$. Since by (c) this history is equal to $f^{(k-1)}\left(H^{(k-1)}\right)$, we conclude from (b) that $o p_{1}$ precedes $o p_{2}$ in $f^{(k-1)}(H)$. But then by (d), this must also be the case in $f^{(k)}(H)$ - a contradiction.

Now assume that not both of $o p_{1}$ and $o p_{2}$ occur in $f^{(k-1)}(H)$. Then by $(\mathrm{d})$ and the assumption that $o p_{2}$ precedes $o p_{1}$ in $f^{(k)}(H)$, we know that $o p_{1}$ does not appear in $f^{(k-1)}(H)$. Hence, according to (3) we can write $f^{(k)}(H)=f^{(k-1)}(H) \circ \lambda$ and $o p_{1}$ appears in $\lambda$. By the induction hypothesis for (b), $o p_{1}$ does not complete during $H^{(k-1)}$. From the assumption $o p_{1} \prec_{H} o p_{2}$, it follows that $o p_{2}$ does not get invoked during $H^{(k-1)}$. Hence, op 2 cannot occur in $f^{(k-1)}(H)=f^{(k-1)}\left(H^{(k-1)}\right)$ either or else $f^{(k-1)}$ would violate (b). Therefore, $o p_{1}$ and $o p_{2}$ must both have events in $\lambda$. By construction, $\lambda$ is a sub-history of $\Gamma\left(H \| O_{i}\right)$ for one object $O_{i}$. Hence, $o p_{1}$ and $o p_{2}$ are both operations on the same object $O_{i}$ and so $o p_{1} \prec_{H \mid O_{i}} o p_{2}$. On the other hand, by the assumption that $o p_{2}$ precedes $o p_{1}$ in $f^{(k)}(H)$ and since by (a) $f^{(k)}(H) \mid O_{i}=f_{i}\left(H^{(k)} \| O_{i}\right)$ and since $f_{i}$ satisfies $(\mathrm{P}), o p_{2}$ occurs before $o p_{1}$ in $f_{i}\left(H \| O_{i}\right)$. But then $f_{i}\left(H \| O_{i}\right)$ is not a linearization of $H \| O_{i}$, contradicting the assumption that $f_{i}$ is a strong linearization function for $\mathcal{H}_{i}$.

So we have proved that the functions $f^{(k)}$ satisfy properties (a) through (d).

The final step of the proof is to use the functions $f^{(k)}$ to define the function $f$ so that it is a strong linearization function for $\mathcal{H}$ (which may contain infinite histories). By property (d), for $k \geq 0$, there is a sequence $\zeta^{(k)}(H)$ satisfying $f^{(k)}(H) \circ \zeta^{(k)}(H)=f^{(k+1)}(H)$. We use this sequence to define $f$ as follows:

$$
f(H)= \begin{cases}f^{(|H|)}(H) & \text { if }|H| \text { is finite, and } \\ f^{(0)}(H) \circ \zeta^{(0)}(H) \circ \zeta^{(1)}(H) \circ \zeta^{(2)}(H) \circ \cdots & \text { if }|H|=\infty .\end{cases}
$$

(Note that if $|H|$ is finite, then $\left.f(H)=f^{(0)}(H) \circ \zeta^{(1)}(H) \circ \cdots \circ \zeta^{(|H|)}(H)\right)$.)

It now remains to confirm that $f$ satisfies properties $(\mathrm{L})$ and $(\mathrm{P})$ of Definition 4.1 .

If $G$ is a prefix of length $\ell$ of some history $H \in \mathcal{H}$, then

$$
f(G)=f^{(\ell)}(G) \stackrel{(c)}{=} f^{(\ell)}(H)=f^{(0)}(H) \circ \zeta^{(0)}(H) \circ \cdots \circ \zeta^{(\ell)}(H)
$$

is a prefix of $f(H)$. Hence, $f$ satisfies property $(\mathrm{P})$.

To show that $f$ satisfies property $(\mathrm{L})$ consider an arbitrary history $H \in \mathcal{H}$. First note that every operation op that completes in $\Gamma(H)$ completes in $f(H)$, too: Let $j$ be the position of the response of $o p$ in $H$. Then by (b) and (c), $f^{(j)}(H)=f^{(j)}\left(H^{(j)}\right)$ is a linearization of $H^{(j)}$. By (d), $f^{(j)}(H)$ is a prefix of $f(H)$ that contains op, since op completes in $\Gamma\left(H^{(j)}\right)$.

Now suppose that $f(H)$ is not a linearization of $\Gamma(H)$. Then there is a finite prefix $G^{\prime}$ of $f(H)$, such that either $\prec_{G^{\prime}}$ is not compatible with $\prec_{H}$, or $G^{\prime}$ is not valid. Let $k$ be an arbitrary large enough integer such that $G^{\prime}$ is a prefix of $f^{(k)}(H)$. By (c), $f^{(k)}(H)=f^{(k)}\left(H^{(k)}\right)$, and so by (b), $f^{(k)}(H)$ extends $\prec_{H^{(k)}}$ and is valid. Clearly, the same is true for every prefix of $f\left(H^{(k)}\right)$. Hence, $G^{\prime}$ is valid and extends $\prec_{H^{(k)}}$, and so $G^{\prime}$ also is compatible with $\prec_{H}$. We conclude that $f(H)$ is a linearization of $H$.

\subsection{Proof that Strong Linearizability is Sufficient for the Strong Adversary.}

We now have the tools to prove the core theorem concerning correctness under the strong adversary.

Proof of Theorem 4.6. Let $\mathcal{H}^{\prime}=\left\{H_{\mathcal{M}^{\prime}, \mathcal{A}^{\prime}, \vec{c}} \mid \vec{c} \in \Omega^{\infty}\right\}$. By Theorem 4.9, since all object implementations are strongly linearizable, $\mathcal{H}^{\prime}$ is strongly linearizable. By Lemma 4.8, there is a normalized 
strong linearization function, say $f$, for $\mathcal{H}^{\prime}$. For each coin flip vector $\vec{c}$, let $H_{\vec{c}}^{\prime}=H_{\mathcal{M}^{\prime}, \mathcal{A}^{\prime}, \vec{c}}$ and $H_{\vec{c}}=f\left(H_{\vec{c}}^{\prime}\right)$. The set of all pairs $\left(\vec{c}, H_{\vec{c}}\right)$ with $\vec{c} \in \Omega^{\infty}$ defines an adversary $\mathcal{A}$, where $\mathcal{A}(\vec{c})$ is the schedule observed by $H_{\vec{c}}$. Then $H_{\vec{c}}=H_{\mathcal{M}, \mathcal{A}, \vec{c}}$ for all $\vec{c} \in \Omega^{\infty}$, and $H_{\vec{c}}$ and $\Gamma\left(H_{\vec{c}}^{\prime}\right)$ have a common linearization (namely $\left.H_{\vec{c}}\right)$. Hence, $(\mathcal{M}, \mathcal{A})$ and $\left(\mathcal{M}^{\prime}, \mathcal{A}^{\prime}\right)$ are equivalent.

It remains to prove that $\mathcal{A}$ is strong for $\mathcal{M}$. That is, for any two coin flip vectors, $\vec{c}=\left(c_{1}, c_{2}, \ldots\right)$ and $\vec{d}=\left(d_{1}, d_{2}, \ldots\right), c_{i}=d_{i}$ for $0 \leq i \leq k$ implies $H_{\vec{c}}[k+1]=H_{\vec{d}}[k+1]$.

Since $\mathcal{A}^{\prime}$ is a strong adversary, it follows that $H_{\vec{c}}^{\prime}[k+1]=H_{\vec{d}}^{\prime}[k+1]$. If $H_{\vec{c}}^{\prime}$ contains fewer than $k+1$ flip operations, then $H_{\vec{c}}^{\prime}=H_{\vec{c}}^{\prime}[k+1]=H_{\vec{d}}^{\prime}[k+1]=H_{\vec{d}}^{\prime}$, and so the claim holds.

Now suppose that $H_{\vec{c}}^{\prime}$ contains at least $k+1$ flip operations. Let $c f$ be the $(k+1)$-st flip in $H_{\vec{c}}^{\prime}$ and $i n v(f l)$ its invocation. Since $H_{\vec{c}}$ is a linearization of $\Gamma\left(H_{\vec{c}}^{\prime}\right)$, and since coin flips are atomic, the $(k+1)$-st flip operation in $H_{\vec{c}}$ is also $c f$, and $i n v(f l)$ is the last step in $H_{\vec{c}}[k+1]$.

Let $G^{\prime}$ and $G$ be the prefixes of $H_{\vec{c}}^{\prime}[k+1]$ and $H_{\vec{c}}[k+1]$, respectively, each of which ends with the last step preceding $i n v(f l)$. Since $G^{\prime}$ is a prefix of $H_{\vec{c}}^{\prime}$, property (P) for $f$ (see Definition 4.1) ensures that $f\left(G^{\prime}\right)$ is a prefix of $f\left(H_{\vec{c}}^{\prime}\right)=H_{\vec{c}}$. Thus $G$ and $f\left(G^{\prime}\right)$ are both prefixes of $H_{\vec{c}}$. We will now show that the last operation of $G$ is in $f\left(G^{\prime}\right)$ and the last operation of $f\left(G^{\prime}\right)$ is in $G$, from which it follows that $f\left(G^{\prime}\right)=G$.

Let $o p$ be the last operation in $G$. Then in $H_{\vec{c}}$, operation op immediately precedes the flip operation $c f$. Consequently, property (N) (see Definition 4.7) ensures that $o p \prec_{H_{\vec{c}}^{\prime}} c f$, and so op completes during $G^{\prime}$. Since $f\left(G^{\prime}\right)$ is a linearization of $G^{\prime}$, op appears in $f\left(G^{\prime}\right)$ as well.

Now consider the last operation $o p^{\prime}$ in $f\left(G^{\prime}\right)$. Since $f$ has property $(\mathrm{P})$ and coin flips appear atomic, $f\left(G^{\prime}\right)$ is a prefix of $H=f\left(G^{\prime} \circ i n v(c f) \circ r s p(c f)\right)$. There can be no operation $o p^{\prime \prime}$ between $o p^{\prime}$ and $c f$ in $H$, because if there were, $o p^{\prime \prime}$ would not complete in $f\left(G^{\prime}\right)$ and thus could not satisfy $o p^{\prime \prime} \prec_{H_{\vec{c}}^{\prime}} c f$, contradicting property $(\mathrm{N})$ for $f$. Thus, $o p^{\prime}$ immediately precedes $c f$ in $H$, and hence also in $f\left(H_{\vec{c}}^{\prime}\right)$. Then by Definition 4.7, $o p^{\prime} \prec_{H_{\vec{c}}^{\prime}} c f$. Therefore, $o p^{\prime}$ precedes $c f$ also in $f\left(H_{\vec{c}}^{\prime}\right)=H_{\vec{c}}$ and so $o p^{\prime}$ appears in $G$.

Thus, we conclude that $f\left(G^{\prime}\right)=G$, and hence $f\left(G^{\prime}\right) \circ \operatorname{inv}(f l)=G \circ \operatorname{inv}(f l)=H_{\vec{c}}[k+1]$. By symmetry we also have $f\left(G^{\prime}\right) \circ i n v(f l)=H_{\vec{d}}[k+1]$, which completes the proof.

\subsection{Timed Executions, Linearization Points, and Composability}

A timed execution is a (possibly infinite) sequence of pairs $E=\left(\left(s_{1}, t_{1}\right),\left(s_{2}, t_{2}\right), \ldots\right)$, where each $s_{i}$ is an invocation or response step and each $t_{i}$ is a number in $\mathbb{R}$ satisfying

- $t_{1} \leq t_{2} \leq \ldots$, and

- if $t_{i}=t_{i+1}$, then $s_{i+1}$ is the response of an (atomic) operation with invocation $s_{i}$.

The timed execution $E$ corresponds to a history $H(E)=\left(s_{1}, s_{2}, \ldots\right)$ together with a timing function $t_{E}:\left\{s_{1}, s_{2}, \ldots\right\} \rightarrow \mathbb{R}, t_{E}\left(s_{i}\right)=t_{i}$. We say that step $s_{i}$ occurs at time $t\left(s_{1}\right)$ in execution $E$. If an operation $o p$ is atomic in $H(E)$, then we say that op occurs atomically at time $t_{E}(i n v(o p))$. Every operation $o p$ of the timed execution $E$ can be associated with an interval $I_{E}(o p) \subseteq \mathbb{R}$, where $I_{E}(o p)=\left[t_{E}(i n v(o p)), t_{E}(r s p(o p))\right]$, if $r s p(o p)$ occurs in $E$, and $I_{E}(o p)=\left[t_{E}(i n v(o p)), \infty\right]$, otherwise.

For a timed execution $E=\left(\left(s_{i}, t_{i}\right)\right)_{i=1,2, \ldots}$ let $\Gamma(E)$ denote the interpretation of $E$, i.e., $\Gamma(E)$ is the timed execution that contains only the pairs $\left(s_{j}, t_{j}\right)$, where $s_{j} \in \Gamma(H(E))$. Define $\Phi(H)$ to be the set of operations whose invocations occur in history $H$, and for a timed execution $E$ let $\Phi(E)=\Phi(H(E))$. 
Now consider an arbitrary timed execution $E$ and the corresponding history $H=H(E)$. It is well-known that a valid sequential history $L$ is a linearization of $\Gamma(H)$ if and only if every operation $o p \in \Phi(\Gamma(E))$ can be mapped to a point $p t(o p) \in \mathbb{R} \cup\{\infty\}$, such that for any op $\in \Phi(\Gamma(E))$ and any distinct $o p_{1}, o p_{2} \in \Phi(L)$ :

(a) $p t(o p) \in I_{E}(o p)$, and

(b) if $o p_{1} \preceq_{L} o p_{2}$, then $p t\left(o p_{1}\right)<p t\left(o p_{2}\right)$.

If $p t: \Phi(\Gamma(E)) \rightarrow \mathbb{R} \cup\{\infty\}$ is a mapping satisfying both, (a) and (b), then we say that $p t$ maps the operations op $\in \Phi(\Gamma(E))$ to their linearization points. Note that by (a) all linearization points $p t(o p)$, with $p t(o p)<\infty$, are distinct. If $p t$ maps the operations in $\Gamma(E)$ to their linearization points, then we denote by $L(E, p t)$ the timed execution, where every operation op $\in \Phi(E)$, op $<\infty$, occurs atomically at time $p t(o p)$.

Two timed executions $E, E^{\prime}$ are isomorphic, if $H(E)=H\left(E^{\prime}\right)$. For a set $\mathcal{E}$ of executions, let close $(\mathcal{E})$ the set of all executions $E^{\prime}$ that are isomorphic to a prefix in $\mathcal{E}$.

Definition 4.11. Let $\mathcal{E}$ be a set of timed executions and for every execution $E \in$ close $(\mathcal{E})$ let a mapping $p t_{E}: \Phi(\Gamma(E)) \rightarrow \mathbb{R} \cup\{\infty\}$ be given. We say that the mappings $p t_{E}, E \in$ close $(\mathcal{E})$, map the operations of $E$ to their strong linearization points, if for all $D, E \in \operatorname{close}(\mathcal{E})$

$\left(L^{\prime}\right) p t_{E}$ maps the operations in $\Gamma(E)$ to their linearization points, and

$\left(P^{\prime}\right)$ if $D$ is a prefix of $E$ then $L\left(D, p t_{D}\right)$ is a prefix of $L\left(E, p t_{E}\right)$.

In this case, $\mathcal{E}$ is called strongly linearizable.

It is immediate that we get the following characterization of strong linearizability of sets of histories:

Lemma 4.12. Let $\mathcal{E}$ be a set of timed executions and $\mathcal{H}=\{H(E) \mid E \in \mathcal{E}\}$. Then $\mathcal{H}$ is strongly linearizable if and only if $\mathcal{E}$ is strongly linearizable.

Proof. Assume w.l.o.g. that $\mathcal{E}=\operatorname{close}(\mathcal{E})$; thus $\mathcal{H}$ is prefix-closed. First suppose that $\mathcal{E}$ is strongly linearizable, i.e., there exist mappings $p t_{E}, E \in \mathcal{E}$, that map the operations in $E$ to their strong linearization points. For each history $H \in \mathcal{H}$, let $E_{H}$ be the timed execution with $H\left(E_{H}\right)=H$, where the $i$-th step of $H$ occurs at time $i$. (Such an execution $E_{H}$ exists because $\mathcal{E}=\operatorname{close}(\mathcal{E})$ is by definition closed under isomorphism.) Then $E_{H} \in \mathcal{E}$ and we can define $f(H)=H\left(L\left(E_{H}, p t_{E_{H}}\right)\right)$. By $\left(L^{\prime}\right), f(H)$ is a linearization of $\Gamma\left(H\left(E_{H}\right)\right)=\Gamma(H)$. Moreover, if $G$ is a prefix of $H$, then by construction $E_{G}$ is a prefix of $E_{H}$, and so from $\left(P^{\prime}\right)$ we immediately get that $f(G)$ is a prefix of $f(H)$. Hence, $f$ is a strong linearization function for $\mathcal{H}$.

Now suppose that $\mathcal{H}$ is strongly linearizable and let $f$ be a strong linearization function of $\mathcal{H}$. Consider an arbitrary timed execution $E \in \mathcal{E}$, let $H=H(E)$ be the corresponding history, and let $t_{E}$ be the corresponding timing function. (I.e., if $E=\left(\left(s_{1}, t_{1}\right),\left(s_{2}, t_{2}\right), \ldots\right)$, then $t_{E}\left(s_{i}\right)=t_{i}$.) Further, let $k=|f(H)| \in \mathbb{N} \cup\{0, \infty\}$, and let $o p_{i}, 1 \leq i \leq k$, be the $i$-th operation in $f(H)$. Finally, for any point time $t \in \mathbb{R}$ let $T^{*}(t)=\left(t+t^{\prime}\right) / 2$, where $t^{\prime}>t$ is the point in time of the first step that occurs in $E$ after time $t$, and $T^{*}(t)=t+1$ if no step occurs in $E$ after point $t$.

We inductively define

$$
\begin{aligned}
& p t_{E}\left(o p_{1}\right)=t_{E}\left(i n v\left(o p_{1}\right)\right), \quad \text { and } \\
& p t_{E}\left(o p_{i}\right)=\max \left\{t_{E}\left(i n v\left(o p_{i}\right)\right), T^{*}\left(p t_{E}\left(o p_{i-1}\right)\right)\right\} \quad \text { for } 1<i \leq k .
\end{aligned}
$$


For every operation $o p \in \Phi(\Gamma(E))-\Phi(f(H))$ we define $p t_{E}(o p)=\infty$.

We first prove property $\left(L^{\prime}\right)$. From the definition of $T^{*}$ we immediately have $p t_{E}\left(o p_{i+1}\right)>$ $p t_{E}\left(o p_{i}\right)$ for all $1 \leq i \leq k$. Thus, it suffices to show that $p t_{E}\left(o p_{i}\right) \in I_{E}\left(o p_{i}\right)$ for $1 \leq i \leq k$. Since by construction $t_{E}\left(i n v\left(o p_{i}\right)\right) \leq p t_{E}\left(o p_{i}\right)$, we only have to show that $p t_{E}\left(o p_{i}\right) \leq t_{E}\left(r s p\left(o p_{i}\right)\right)$. We prove by induction on $i$ for all $1 \leq i \leq k$ the following statement: $p t_{E}\left(o p_{i}\right) \leq t_{E}\left(r s p\left(o p_{i}\right)\right.$ and $p t_{E}\left(o p_{i}\right)<t_{E}\left(r s p\left(o p_{j}\right)\right)$ for all $i \leq j \leq k$. For $i=1$ the statement is true since $p t_{E}\left(o p_{1}\right)=$ $t_{E}\left(i n v\left(o p_{1}\right)\right)$ and any response step in $E$ occurs no earlier than $t_{E}\left(i n v\left(o p_{1}\right)\right)$, and only $r s p\left(o p_{1}\right)$ can occur at the same time as $i n v\left(o p_{1}\right)$ (if $o p_{1}$ is atomic in $E$ ). Thus, let $1<i \leq j \leq k$. Since $f(H)$ is a linearization of $\Gamma(H(E))$, we know that in $E$ the invocation of $o p_{i}$ occurs no later than the response of $o p_{j}$, and they can occur at the same time only if $i=j$ (and $o p_{i}$ is atomic). Therefore, $t_{E}\left(i n v\left(o p_{i}\right)\right) \leq t_{E}\left(r s p\left(o p_{j}\right)\right)$, and equality holds only if $i=j$. Hence, if $p t_{E}\left(o p_{i}\right)=t_{E}\left(i n v\left(o p_{i}\right)\right)$, we are done. So assume that $t_{E}\left(i n v\left(o p_{i-1}\right)\right)<p t_{E}\left(o p_{i}\right)$. By the induction hypothesis, and since $j>i-1$, we have $p t_{E}\left(o p_{i-1}\right)<t_{E}\left(r s p\left(o p_{j}\right)\right)$, and then from the definitions of $p t_{E}$ and $T^{*}$, we get

$$
p t_{E}\left(o p_{i}\right)=T^{*}\left(p t_{E}\left(o p_{i-1}\right)\right) \leq \frac{p t_{E}\left(o p_{i-1}\right)+t_{E}\left(r s p\left(o p_{j}\right)\right)}{2}<t_{E}\left(r s p\left(o p_{j}\right)\right) .
$$

We now show $\left(P^{\prime}\right)$. Consider two arbitrary timed executions $D, E \in \mathcal{E}$ such that $D$ is a prefix of $E$. Let $f(H(D))=\left(o p_{1}, \ldots, o p_{k}\right)$. Since $f$ is prefix preserving, $o p_{1}, \ldots, o p_{k}$ are the first $k$ operations in $f(H(E))$. It is immediate from the inductive construction that $p t_{D}\left(o p_{i}\right)=p t_{E}\left(o p_{i}\right)$ for $1 \leq i \leq k$, and $p t_{E}(o p)>p t_{E}\left(o p_{k}\right)=p t_{D}(o p)$ for all operations op $\in f(H(E))-\left\{o p_{1}, \ldots, o p_{k}\right\}$. Thus, $L\left(D, p t_{D}\right)$ is a prefix of $L\left(E, p t_{E}\right)$.

Theorem 4.13 (Composability). Let $\mathcal{B}$ and $\mathcal{B}^{\prime}$ be disjoint sets of base objects. Further, let $O$ be a strongly linearizable implementation of some type $T$ that uses only base objects in $\mathcal{B}$. Also, for some atomic object $B \in \mathcal{B}$ let $B^{\prime}$ be an object of the same type as $B$ that is implemented from objects in $\mathcal{B}^{\prime}$. Then, the implemented object $O^{\prime}$ obtained from $O$ by replacing object $B$ with $B^{\prime}$ is a strongly linearizable implementation of type $T$ that uses atomic base objects from the set $(\mathcal{B}-\{B\}) \cup \mathcal{B}^{\prime}$.

Proof. Let $\mathcal{E}_{O^{\prime}}, \mathcal{E}_{O}$, and $\mathcal{E}_{B^{\prime}}$ be the set of all timed executions that can occur if processes execute operations on $O^{\prime}, O$, and $B^{\prime}$, respectively. By the assumption, $\mathcal{E}_{O}$ and $\mathcal{E}_{B^{\prime}}$ are strongly linearizable. Let $p t_{E}^{\prime}$ for $E \in \mathcal{E}_{B^{\prime}}$ and $p t_{D}^{\prime \prime}$ for $D \in \mathcal{E}_{O}$ denote mappings that map executions in $\mathcal{E}_{B^{\prime}}$ and $\mathcal{E}_{O}$, respectively, to their strong linearization points.

Now consider an arbitrary timed execution $E=\left(\left(s_{1}, t_{1}\right), \ldots,\right) \in \mathcal{E}_{O^{\prime}}$. Then $E \mid B^{\prime} \in \mathcal{E}_{\mathcal{B}^{\prime}}$, and thus every operation $o p \in E \mid B^{\prime}$ is associated with a strong linearization point $p t_{E \mid B^{\prime}}^{\prime}(o p)$. For every such operation op executed by some process $p$ during $E$, we do the following: We remove all steps from $E$, that a process $p$ executes during the interval that starts with $i n v(o p)$ and ends with $r s p(o p)$, and if $p t_{E \mid B^{\prime}}^{\prime}(o p)<\infty$, then we replace those steps with the atomic operation op at time $p t_{E \mid B^{\prime}}^{\prime}(o p)$. Thus, in the resulting execution, $\alpha(E)$, all operations op on object $B^{\prime}$ are atomic. Also, $\alpha(E) \mid B^{\prime}=L\left(E \mid B^{\prime}, p t_{E \mid B^{\prime}}^{\prime}\right)$, and so $H\left(\alpha(E) \mid B^{\prime}\right)$ is a linearization of $\Gamma\left(E \mid B^{\prime}\right)$. Moreover, all steps that are not on $B^{\prime}$ occur at exactly the same time in $E$ as in $\alpha(E)$, and very process executes its steps in program order.

Thus, $\alpha(E)$ is a timed execution in $\mathcal{E}_{O}$, and we can define the mapping $p t_{E}: \Phi(\Gamma(E)) \rightarrow \mathbb{R} \cup\{\infty\}$ by

$$
p t_{E}(o p)=p t_{\alpha(E)}^{\prime \prime}(o p)
$$


All invocation and response steps on $O$ appear at exactly the same time in $E$ as in $\alpha(E)$, i.e., $\Gamma(E)=\Gamma(\alpha(E))$. Since $p t_{\alpha(E)}^{\prime \prime}(o p)$ maps the operations in $\Gamma(\alpha(E))$ to their linearization points, it is obvious that $p t_{E}$ satisfies property $\left(L^{\prime}\right)$.

It remains to show property $\left(P^{\prime}\right)$. Let $D, E \in \mathcal{E}_{O^{\prime}}$, and let $D$ be a prefix of $E$. Then $D \mid B^{\prime}$ is a prefix of $E \mid B^{\prime}$, and since $p t_{D \mid B^{\prime}}^{\prime}$ and $p t_{E \mid B^{\prime}}^{\prime}$ map the operations in $D \mid B^{\prime}$ and $E \mid B^{\prime}$, respectively, to their strong linearization points, $\alpha(D) \mid B^{\prime}=L\left(D \mid B^{\prime}, p t_{D \mid B^{\prime}}^{\prime}\right)$ is a prefix of $\alpha(E) \mid B^{\prime}=L\left(E \mid B^{\prime}, p t_{E \mid B^{\prime}}^{\prime}\right)$. As a consequence, $\alpha(D)$ is a prefix of $\alpha(E)$, and so $L\left(\alpha(D), p t_{\alpha(D)}^{\prime \prime}\right)=L\left(D, p t_{D}\right)$ is a prefix of $L\left(\alpha(E), p t_{\alpha(E)}^{\prime \prime}\right)=L\left(E, p t_{E}\right)$.

\subsection{Which Linearizable Implementations are Strongly Linearizable?}

Many linearizable implementations of shared objects from the literature are actually strongly linearizable, and hence can be used safely in randomized algorithms, in place of their atomic counterparts. We can identify some of these by examining the proof of linearizability of the implementation. Such a proof typically follows one of a few general approaches. Let $O$ be the implemented object and let $H$ be a history over $O$.

In one proof technique, for each operation op on $O$ a unique "linearization point" $p t(o p)$ is assigned, which is a shared memory operation that occurs during the execution of operation $o p$. A sequential history $S$ is formed by ordering the operations on $O$ in $H$ so that $o p_{1}$ precedes $o p_{2}$ in $S$ if (and only if) $p t\left(o p_{1}\right) \prec_{H} p t\left(o p_{2}\right)$. The construction of $S$ guarantees agreement with the "happens before" order of operations on $O$ in $H$, and so the proof obligation for linearizability is only to show that $S$ is valid for $O$. If the linearization points are chosen so that the mapping from $H$ to $S$ also satisfies property (P) (see Definition 4.1), then the implementation is strongly linearizable. Property $(\mathrm{P})$ will hold, for example, if, for any shared memory operation $o p^{\prime}$ in the software method that simulates $o p$, by the time $o p^{\prime}$ is executed it is determined whether or not $o p^{\prime}$ is the linearization point for $o p$. This holds, for instance, if $p t(o p)$ is mapped statically to a particular pseudo-code statement (that performs exactly one shared memory operation), irrespective of the schedule.

Several published implementations admit such proofs of strong linearizability, for example an obstruction-free double-ended queue [HLM03] and a terminating Compare-And-Swap implementation from atomic registers [GHHW07]. The practical constructions of sets and lists such as the "FineList", "OptimisticList", and "LazyList" described by Herlihy and Shavit [HS08] use finegrained locking and are strongly linearizable for the same reason. For non-blocking constructions of sets, there typically remains a point in the code (often a strong synchronization operation) associated with each implemented "insert" or "delete" operation, which allows the proofs of linearizability to extend to proofs of strong linearizability (see for example the LockFreeList in [HS08]).

Many universal constructions also fall into this category because they force operations to take effect one at a time 3 For example, Herlihy's universal construction for wait-free shared objects [Her91] is strongly linearizable. In this construction, each operation applied on the shared object is represented using a "cell" data structure. Processes cooperate to thread cells onto a list; they reach agreement on the successor of each cell through a consensus object for that cell. The linearization corresponding to the total order of the cells in this list defines a strong linearization 4

\footnotetext{
${ }^{3}$ In that case the linearization order follows easily from the algorithm and a formal proof of linearizability is often not given.

${ }^{4}$ If the implementation is non-deterministic and the operation corresponding to the last cell in the list is not
} 
Similarly, any implementation of a shared object obtained by wrapping a mutex around a sequential implementation is strongly linearizable. Combining this observation with known implementations of mutual exclusion using only safe registers, yields strongly linearizable implementations of any shared object using only safe registers. Strongly linearizable implementations with $\varnothing(1)$ Remote Memory Reference (RMR) complexity are also possible using a queue-based mutex, which can be constructed using atomic registers and certain Read-Modify-Write primitives such as Fetch-And-Add or Fetch-And-Store.

In the second general proof technique, the operations in a history $H$ are first ordered somehow into a valid sequence $S$, and the proof obligation is to show that $S$ is consistent with the "happens before" order of $H$. Such proofs typically do not directly translate to proofs of strong linearizability, and the corresponding implementations are frequently not strongly linearizable. Two such examples are in the introduction. Additional examples of implementations in this category include other "textbook" wait-free constructions of strong atomic registers from weaker ones. For instance, any strong adversary has less power against a randomized algorithm using atomic MRSW registers, than a particular weak adversary has against the same algorithm when the MRSW registers are replaced with Israeli and Li's linearizable construction from SRSW atomic registers [IL93. Similarly, any strong adversary has less power against a randomized algorithm using atomic MRSW registers, than a weak adversary has against the same algorithm when the MRMW registers are replaced with Vitanyi and Awerbuch's linearizable construction from MRSW atomic registers [VA86. The impasse is not just for register-like constructions. Herlihy and Wing [HW90] provide a linearizable implementation of a queue object using some read-modify-write objects where the ENQ operation but not the DEQ operation is wait-free. There is no strong linearization even for the subset of histories that occur when DEQ is constrained to be atomic. (Examples of all these situations are included Appendix A, )

\subsubsection{Strong Linearizability of CAS Implementation from Atomic Registers}

We now sketch a proof of strong linearizability for the RMR-efficient implementation of CompareAnd-Swap from atomic registers GHHW07. First, we describe briefly how this implementation works, letting $O$ denote the implemented CAS object. The basic implementation supports READ and CAS operations, and is presented in a simplified format in Figure3. (A WRITE operation can be implemented in a strongly linearizable way using similar techniques [Gol10].) The implementation records the state of $O$ using data structures called blocks. Each block contains a variable $V a l$ that records a state (i.e., value) of $O$. (For any block, $\mathrm{Val}$ inside that block is written at most once.) The state stored in block $b$ is denoted $b . V a l$. The block that stores the latest state of $O$ is called the current block, and its address is stored in a shared register Cur. An operation that does not change the state of $O$ simply reads $C$ ur to identify the current block, and reads the state of $O$ from a shared variable in that block (lines 144 and lines 18 19). An operation that causes a non-trivial state change allocates a new block (line 8), writes the new state to that block (line 9), and writes the address of that block to Cur (line 10). At the heart of the implementation lies a mechanism for efficient synchronization among operations that attempt to apply non-trivial state changes concurrently (line 6). This mechanism ensures that at most one such operation succeeds

and the others instead apply trivial state transitions. An efficient signaling mechanism is also used between the leader and losers, as we explain shortly (lines 11, 14).

complete, then that operation is dropped from the linearization because its response may not be uniquely determined. 


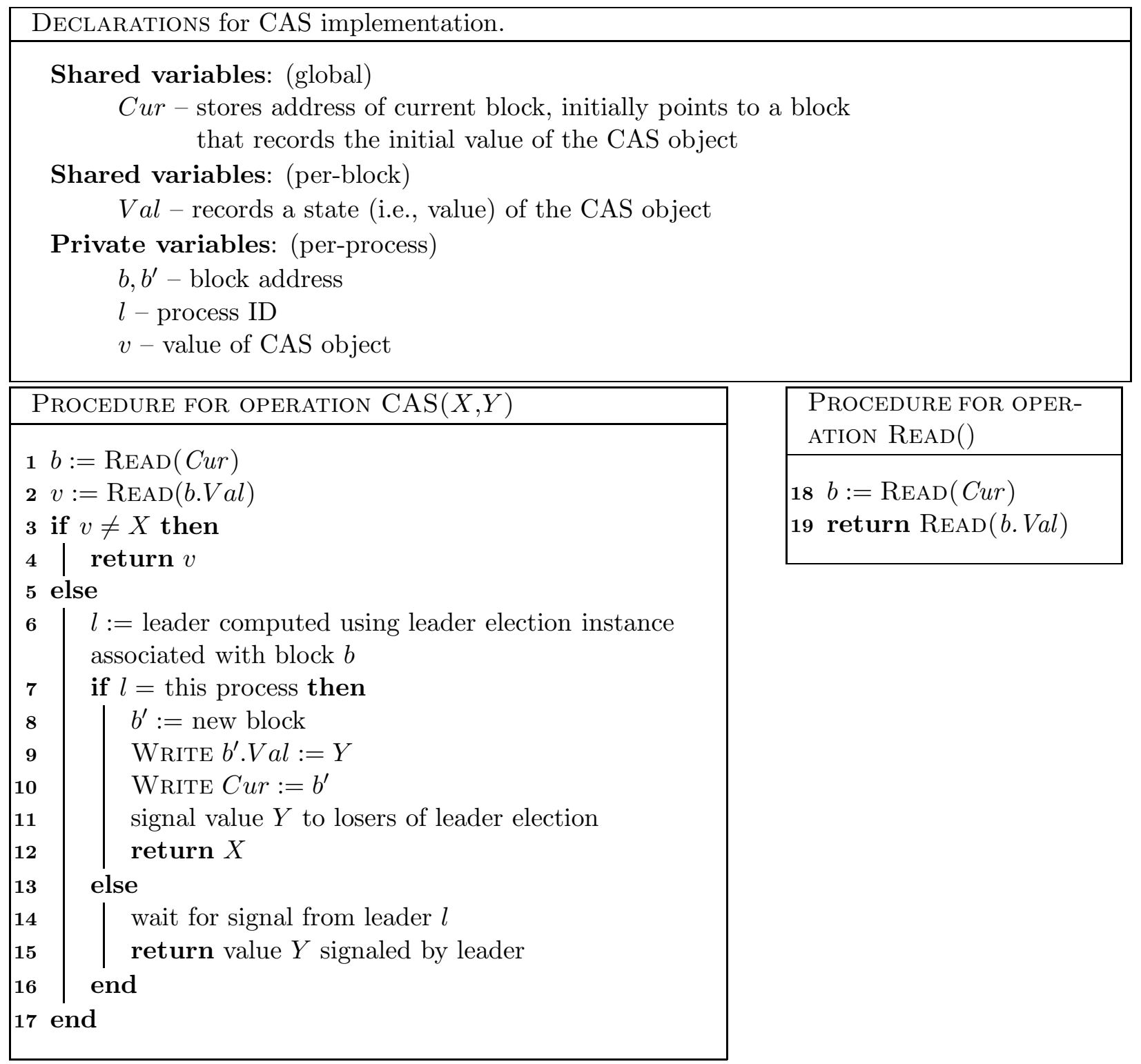

Figure 3: Implementation of CAS from reads and writes. 
We now illustrate how the implementation works with a short example. Let $s_{0}, s_{1}, s_{2}, \ldots$ denote the sequence of states $O$ takes on (ignoring trivial state transitions), and let $b_{0}, b_{1}, b_{2}, \ldots$ denote the blocks that store these states. (The state of a CAS object is simply the value it stores.) Suppose that the current block is $C u r=b_{2}$ and processes $p_{1}, p_{2}, p_{3}, p_{4}$ invoke the following operations concurrently: $p_{1}, p_{2}$ and $p_{3}$ invoke $\operatorname{CAS}\left(s_{2}, X_{1}\right), \operatorname{CAS}\left(s_{2}, X_{2}\right)$ and $\operatorname{CAS}\left(s_{2}, X_{3}\right)$ respectively, where $s_{2} \notin\left\{X_{1}, X_{2}, X_{3}\right\}$; and $p_{4}$ invokes $\operatorname{CAS}(Y, Z)$ for some $Y \notin\left\{s_{2}, X_{1}, X_{2}, X_{3}\right\}$ and arbitrary $Z$. Process $p_{4}$ 's operation is dealt with easily; upon discovering that the state $s_{2}$ stored in block $C u r=b_{2}$ is different from $Y, p_{4}$ 's $\operatorname{CAS}(Y, Z)$ does not change the state of $O$ and returns $s_{2}$ (line (4). In contrast, each of $p_{1}, p_{2}, p_{3}$ has a chance to change the state of $O$, although only one of them may actually do so. Thus, $p_{1}, p_{2}, p_{3}$ elect a leader, say $p_{2}$, which allocates a new block $b$ and records $X_{2}$ in it (lines 8 9). (A separate "instance" of leader election is used to synchronize each non-trivial state change, and we associate such instances with blocks on a one-to-one basis. In this example, $p_{1}, p_{2}, p_{3}$ use the instance associated with block $b_{2}$.) Process $p_{2}$ then sets $C u r=b$ (line 10) and its $\operatorname{CAS}\left(s_{2}, X_{2}\right)$ returns $s_{2}$ (line 12). Thus, $b_{3}=b$ and $s_{3}=X_{2}$ hold, and the state of $O$ changes from $s_{2}$ to $s_{3}$. Meanwhile, $p_{1}$ and $p_{3}$ wait until $p_{2}$ has written $C u r$ (line 14), and their operations return $s_{3}$ (line 15). (The linearizability of the implementation depends crucially on $p_{1}$ and $p_{3}$ waiting for $p_{2}$ in this situation.) Thus, their ("failed") CAS operations cause trivial state transitions from $s_{3}$ back to $s_{3}$, and appear to take effect just after $p_{2}$ 's ("successful") CAS.

To show strong linearizability, we will use timed executions (see Section 4.6). For any history $H$ of the implementation, define a corresponding timed execution $E$ where the $i$ 'th step occurs at time $i$. Let $\mathcal{E}$ denote the set of such timed executions. For any $E \in \mathcal{E}$, we define the mapping $p t_{E}: \Phi(\Gamma(E)) \rightarrow \mathbb{R} \cup\{\infty\}$ as follows. If $o p$ is a READ operation that invokes a read of Cur at time $t$, then $p t_{E}(o p)=t$, otherwise $o p$ is pending in $E$ and $p t_{E}(o p)=\infty$. If $o p$ is a $\operatorname{CAS}(X, X)$ operation for some $X$ (i.e., the comparison value is the same as the new value), we treat it just like a READ. If $o p$ is a $\operatorname{CAS}(X, Y)$ operation for some $X$ and $Y \neq X$, then $p t_{E}(o p)$ depends on the execution path of $o p$. If $o p$ is pending and does not read $C u r$ at all, then $p t_{E}(o p)=\infty$. If $o p$ invokes a read of $C u r$ at time $t$, and the state of $\varnothing$ in the block read is not $X$, then op is a "failed" CAS and $p t_{E}(o p)=t$. If $o p$ invokes a read of $C u r$ at time $t$, and the state of $\varnothing$ in the block read is $X$, then $o p$ may succeed or fail depending on the outcome of leader election (i.e., the "instance" of leader election associated with the block whose address op reads from $C u r$ ). If the outcome of leader election is not "decided" at the end of $E$ (i.e., different extensions of $E$ may lead to different outcomes), or the outcome is decided but the leader's operation has not yet overwritten Cur, then $p t_{E}(o p)=\infty$. (In this case $o p$ is pending because the implementation ensures that the leader writes Cur before $o p$ terminates, even if $o p$ is not the leader's operation.) Otherwise the write to Cur by the leader is invoked at some time $t$. If $o p$ is the leader's operation then $p t_{E}(o p)=t$. Finally, if $o p$ is not the leader's operation, then $p t_{E}(o p)=t+\varepsilon$ where $0<\varepsilon<1$ is an arbitrary constant unique for each process.

It is straightforward to verify that for each $E \in \mathcal{E}, L\left(E, p t_{E}\right)$ is a linearization of $\Gamma(E)$. Next, consider Definition 4.11. Property $\left(L^{\prime}\right)$ follows easily for any operation op whose linearization point is the time of a step that $o p$ itself takes, which is always between the times of $i n v(o p)$ and $r s p(o p)$. In all remaining cases, the linearization point is of the form $t+\varepsilon$ where $t$ is the time of a step that is not part of $o p$ but nevertheless occurs after $i n v(o p)$ and before $r s p(o p)$. Since the times at which steps occur are integer-valued by construction of $E$, and since $0<\varepsilon<1$, it follows that $t+\varepsilon$ is also between the times of $i n v(o p)$ and $r s p(o p)$, as wanted. For property $\left(P^{\prime}\right)$, consider histories $D, E \in \mathcal{E}$ such that $D$ is a prefix of $E$. It suffices to consider the case when $E$ extends $D$ 
by one step, say $s$ at time $t$, and show that $L\left(D, p t_{D}\right)$ is a prefix of $L\left(E, p t_{E}\right)$. If $s$ is not a step that reads or writes the shared variable $C u r$, then $L\left(E, p t_{E}\right)=L\left(D, p t_{D}\right)$. If $s$ reads $C u r$ and is part of some operation $o p$, then either $p t_{E}(o p)=\infty$ and $L\left(E, p t_{E}\right)=L\left(D, p t_{D}\right)$ (i.e., op is pending and trying to cause a non-trivial state change), or else $L\left(E, p t_{E}\right)$ is an extension of $L\left(D, p t_{D}\right)$ by an invocation/response pair for $o p$ (i.e., op causes a trivial state change). Finally, if $s$ writes $C u r$ and is part of operation $o p$, then $L\left(E, p t_{E}\right)$ is an extension of $L\left(D, p t_{D}\right)$ by an invocation/response pair for $o p$, followed by zero or more invocation/response pairs for operations whose linearization points are of the form $t+\varepsilon, 0<\varepsilon<1$.

\section{$5 \quad$ Weak Adversaries}

In this section we show that it is impossible to strengthen linearizability in a way that limits the power of a weak adversary to influence the result of an execution in the same sense as strong linearizability limits the power of the strong adversary, when implementations are obtained only from atomic registers and load-linked/store-conditional. To prove this, we consider a particular algorithm that uses shared objects of a "strong counter" type. The state of this type is an integer (initially 0) and the operations supported are FETCH\&INC() and FETCH\&DEC(). These operations increment and decrement the counter, respectively, and also return the prior value of the counter. In the executions we will consider, operations will be invoked in such a way that the counter's value is always in $[0, n]$ where $n$ is the maximum number of processes.

In Figure 4, we present a simple algorithm that uses $\sqrt{n}$ strong counters. (Throughout this section we assume that $n$ is a perfect square.) Each process chooses one of the counters uniformly at random, then calls FETCH\&INC(), and finally calls FETCH\&DEC(). Now fix a weak adversary $\mathcal{A}$ and an integer $K_{\max }$. Let $H$ be a random history obtained by a run of LOADBALANCE() scheduled by $\mathcal{A}$. For each process $p \in \mathcal{P}$ let $X_{\mathcal{A}, p}$ be the random variable defined as follows: If during $H$ the maximum point contention 5 is at most $K_{\max }$ and $p$ 's FETCH\&INC() terminates, then $X_{\mathcal{A}, p}$ is the value returned by that FETCH\&INC(). If $p$ 's FETCH\&INC() does not terminate during $H$, or if the point contention exceeds $K_{\text {max }}$, then define $X_{p}=0$. We are interested in the maximum expectation of $X_{\mathcal{A}, p}$ over all processes, i.e.,

$$
\Phi(\mathcal{A}):=\max \left\{\mathrm{E}\left[X_{p . \mathcal{A}}\right] \mid p \in \mathcal{P}\right\}
$$

It is not hard to see that if the FETCH\&INC () operation is atomic, then for all weak adversaries $\mathcal{A}$, $\Phi(\mathcal{A}) \leq K_{\max } / \sqrt{n}$. In particular, if $K_{\max }=\Theta(\sqrt{n})$, then $\Phi(\mathcal{A})=O(1)$.

On the other hand, we will show that if the FETCH\&INC() operations are based on a "natural" terminating (or lock-free) implementation that uses only read, write, and LL/SC operations, then for $K_{\max }=\Theta(\sqrt{n})$ there exists a weak adversary $\mathcal{A}$ such that $\Phi(\mathcal{A})=\Omega\left(K_{\max }\right)=\Omega(\sqrt{n})$. An implementation of a type $\tau$ is natural if each instance $O$ of the implemented object has its own set $\mathcal{B}_{O}$ of base objects such that an operation on the instance $O$ accesses only base objects in $\mathcal{B}_{O}$. Essentially all linearizable implementations of objects are natural, as otherwise the composition of multiple objects would effectively create a single new object.

Strong counters can be implemented with various progress properties from atomic read, write, and LL/SC operations. For example, a deterministic wait-free implementation can be obtained using

\footnotetext{
${ }^{5}$ The maximum point contention is the maximum number of processes that at the same point in time have called LOADBALANCE() but not yet finished that function call, where the maximum is taken over all points in time.
} 
FunCtion LoAdBalance()

Shared data: $\sqrt{n}$ shared strong counters, $F_{0}, \ldots, F_{\sqrt{n}-1}$

1 Choose $i \in\{0, \ldots, \sqrt{n}-1\}$ uniformly at random.

$2 x:=F_{i}$. FETCH\&INC ()

$3 F_{i}$.FETCH\&DEC ()

4 return $x$

Figure 4: The load balancing algorithm.

Herlihy's universal construction [Her91], with consensus objects simulated in a straightforward way from LL/SC. Given only read and write, a deterministic terminating implementation is possible using Yang and Anderson's mutual exclusion algorithm [YA95]. (Alternately, one can simulate LL/SC in the wait-free implementation using read and write [GHHW07], which yields a terminating strong counter implementation from read and write only.) All these implementations are natural.

We now present our main result, stating that a weak adversary gains additional power against algorithm LOADBALANCE no matter how the strong counters are implemented from read, write, and LL/SC.

Theorem 5.1. Let $K_{\max }=\lceil(1+\delta) \sqrt{n}\rceil, \delta>0$. If the algorithm in Figure 4 (LoAdBALANCE) is used with atomic strong counters, then for any weak adversary $\mathcal{A}, \Phi(\mathcal{A})<1+\delta=O(1)$ holds. On the other hand, if the algorithm LOADBALANCE is used with a linearizable, natural, possibly randomized, terminating (or lock-free) implementation of strong counters from atomic read, write, and LL/SC operations, then there exists a weak adversary $\mathcal{A}$, such that $\Phi(\mathcal{A})=\Omega\left(K_{\max }\right)=\Omega(\sqrt{n})$.

The full proof of the theorem is quite complicated and given in the extended version of this paper. Here we sketch the main idea.

Proof sketch. For the upper bound, observe that at the point in time when a process $p$ makes its random choice, the expected value of the counter it chooses is at most $k / \sqrt{n}$, where $k$ is the number of processes currently active. Since the weak adversary cannot intervene between $p$ 's random choice and $p$ 's FETCH\&INC(), that operation's return value is at most $(k-1) / \sqrt{n}$.

For the lower bound, we construct for each process $p$ a weak adversary $\mathcal{A}_{p}$ that tries to "fool" $p$. We fix the coin flips that processes receive arbitrarily. Then we choose one process $p$ at random, use $\mathcal{A}_{p}$ for the scheduling, and obtain that the expected return value of $p$ 's FETCH\&INC () is $\Omega(k)$, where $k$ is the number of processes that randomly chose the same counter as $p$. Also, point contention is at most $k+1$. If coin flips are uniformly random, then $k$ is highly concentrated around $\sqrt{n}$, so with high probability it will be $\Omega(\sqrt{n})$ but also not exceed $K_{\max }$.

So the main goal of the adversary $\mathcal{A}_{p}$ is to make $p$ 's FETCH\&INC() call return a value with expectation $\Omega(k)$. We achieve this as follows. First, we let process $p$ take one step, which reveals the index $i^{*}$ of the counter it is using. Then we schedule each process $q \neq p$, one after the other. If $q$ accesses a different counter than the one chosen by $p$, we let $q$ run solo until it finishes the algorithm (after that $q$ does not contribute to point-contention anymore). If $q$ also chooses the counter with index $i^{*}$, then we let it take exactly one step and then stall it. This way, eventually all $k$ processes that chose the same counter as $p$ are stalled, while all other processes are finished. Moreover, the maximum point contention encountered is at most $k+1$. 
Now we partition the set of stalled processes into three sets. Let $o p_{q}$ be the first (and only) operation process $q$ executed so far. The set $\mathcal{Q}$ contains all processes $q$, where $o p_{q}$ is not a write. The set $\mathcal{V}$ contains all processes $q$, where $o p_{q}$ is a write to a register $R$ to which no other process writes in its first step. Finally, $\mathcal{W}$ is the set of all remaining processes, which write to some register that is also written by another process. Note that the sets $\mathcal{Q}, \mathcal{V}$, and $\mathcal{W}$ are uniquely determined by the first steps executed by all processes, and thus by the coin flips processes use. But they are independent of the choice of $p$.

We make use of a positive correlation between the size of each set, and the probability with which $p$ is from that set. Suppose $\mathcal{Q}$ is large, i.e., $|\mathcal{Q}| \geq k / 3$. Then the probability that $p \in \mathcal{Q}$ is at least $1 / 3$. Moreover, given that $p \in \mathcal{Q}, p$ 's first operation $o p_{p}$ is not a write, and thus leaves no "trace" (note that if it is a SC, then it fails). In this case, we stall $p$ and let all other $k-1$ processes finish their $F_{i^{*}}$.FETCH\&INC() operation. After that $F_{i^{*}}$ has value $k-1$, so that when we finally let $p$ finish its FETCH\&INC(), its return value is $k$.

Now suppose $|\mathcal{V}| \geq k / 3$, so the probability that $p \in \mathcal{V}$ is at least $1 / 3$. If $p \in \mathcal{V}$, we let all processes in $\mathcal{V}$ run in a round-robin fashion in some predetermined order, until they have finished their algorithm. It can be argued that the choice of $p$ among processes in $\mathcal{V}$ has no influence on what processes in $\mathcal{V}$ observe in the resulting execution. Hence, the FETCH\&INC() operations of all processes in $\mathcal{V}$ return distinct values. Given that $p \in \mathcal{V}$ is chosen uniformly at random, $p$ 's FETCH\&INC () return value has an expectation of at least $(|\mathcal{V}|-1) / 2=\Omega(k)$.

Finally, suppose $|\mathcal{W}| \geq k / 3$. If $p \in \mathcal{W}$, then $o p_{p}$ is a write that was overwritten by the operation $o p_{q}$ of some other process $q$ (recall that $p$ 's write was scheduled before any other process took a step). Moreover, since the first operation of all other processes in $\mathcal{V} \cup \mathcal{W}$ is a write, none of these processes can have "seen" $p$ before $p$ was overwritten. Any process that may have seen $p$ cannot have executed a write in its first step, so it didn't become "visible" itself. We let all processes in $\mathcal{V} \cup \mathcal{W}-\{p\}$ finish their FETCH\&INC call by scheduling them in a round-robin fashion. They can only see themselves, and not any process outside of this set, so they will increase the value of the counter to at least $|\mathcal{V} \cup \mathcal{W}| \geq k / 3$. When we run $p$ afterward, its FETCH\&INC() call must return a value of $\Omega(k)$.

For the complete proof of Theorem 5.1 we rely on Theorems 5.2 and 5.3 below.

Theorem 5.2. If Algorithm 4 (LOADBALANCE) is used with atomic strong counters, then for any weak adversary $\mathcal{A}$ and any integer $K_{\max }$

$$
\Phi(\mathcal{A}) \leq \frac{K_{\max }-1}{\sqrt{n}}
$$

Proof. Let $m=\sqrt{n}$ and let $\mathcal{A}$ be an arbitrary weak adversary. Fix an arbitrary process $p$. Since $X_{\mathcal{A}, p}$ is 0 if $K>K_{\max }$, it suffices to show that $\mathrm{E}\left[X_{\mathcal{A}, p} \mid K\right] \leq(K-1) / m$.

Consider the system configuration immediately before $p$ makes its random choice in line 1 . Suppose that in this configuration the counter $F_{j}, 0 \leq j<m$, has value $b_{j}$. Then clearly there are at least $b_{0}+\cdots+b_{m-1}+1$ processes active (including $p$ ). Thus, given $K$, we have $b_{0}+\cdots+b_{m-1}+1 \leq K$. Hence, when $p$ makes its random choice, the expected value of the counter chosen by $p$ is

$$
\sum_{0 \leq j<m} \frac{b_{j}}{m} \leq \frac{K-1}{m} .
$$

Since the adversary cannot intervene between $p$ 's random choice and $p$ 's following FETCH\&INC() operation, the expected return value of that operation is at most $(K-1) / m$, as wanted. 
Next, we consider the case when Algorithm 4 is used with linearizable implementations of strong counters from atomic read, write and LL/SC operations. Our analysis applies to any terminating or lock-free (hence wait-free) implementation of strong counters from read, write and LL/SC that is natural.

Theorem 5.3. Let $K_{\max }=[(1+\delta) \sqrt{n}]$, for some arbitrarily small $\delta>0$. Consider Algorithm 4 (LOADBALANCE) used with a linearizable, natural, possibly randomized, terminating (or lock-free) implementation of strong counters from atomic read, write, and LL/SC operations. Then there exists a weak adversary $\mathcal{A}$, such that

$$
\Phi(\mathcal{A})=\Omega\left(K_{\max }\right)=\Omega(\sqrt{n})
$$

To prove Theorem 5.3 first we specify the adversary $\mathcal{A}$ (in Subsection 5.1 ), and second present the analysis that bounds $\Phi(\mathcal{A})$ from below (Subsection 5.2 ).

For the purpose of the following definition, it is convenient to assume w.l.o.g. that registers store pairs of values, where the second component of such a pair stores which process changed the register last. We can achieve this by having processes write their ID into the second component of the pair with each successful write or SC operation. We say, process $q$ marks the register with its ID. If later on some other process $z \neq q$ writes to the same register, or performs a successful SC operation on that register, $q$ 's mark gets replaced by $z$ 's mark.

We say that in some configuration $C$ process $p$ is visible, if in $C$ some register is marked by $p$. During a history $H$ a process $q$ sees process $p \neq q$, if

- either $q$ reads or performs an LL operation on a register marked by $p$, or

- $q$ executes an SC operation on some register $R$, at a point in time when $R$ is marked by $p$, and after $q$ has executed an LL operation on $R$. (If this happens, then $q$ 's SC operation fails.)

Observation 5.4. If $H$ is a history where no process in $P \subseteq \mathcal{P}$ sees a process in $\bar{P}$, then $H \mid P$ is indistinguishable from $H$ to all processes in $P$.

\subsection{Specification of the Adversary $\mathcal{A}_{p}$}

First we describe for each process $p$ some weak adversary $\mathcal{A}_{p}$ that is trying to "fool" the process $p$ For the analysis we will then choose $p$ at random.

Adversary $\mathcal{A}_{p}, p \in \mathcal{P}$, is defined as follows: We let $p$ take steps until $p$ has performed its first shared memory access (during its FETCH\&INC() operation). Note that this access reveals the index $i^{*}$ of the counter $F_{i^{*}}$ that $p$ chose. After that we stall $p$.

Next we consider each of the remaining processes $q \in \mathcal{P}-\{p\}$, one after the other, in the order of their IDs. We let $q$ take steps until it has executed its first shared memory access and thus revealed its choice of a counter $F_{i_{q}}$. If $i_{q} \neq i^{*}$, we let $q$ run solo, until it has finished its run of LOADBALANCE completely. Otherwise, we stall $q$. This is continued until each processes in $\mathcal{P}$ either has finished its LOADBALANCE algorithm or is stalled.

Let $H^{\prime}$ be the execution obtained so far, and $C$ the system configuration at the end of $H^{\prime}$. Further, let $\mathcal{P}_{j}, 0 \leq j<m$, denote the set of the processes that selected the counter $j$ during $H^{\prime}$. Note that in $H^{\prime}$, all processes in $\mathcal{P}-\mathcal{P}_{i^{*}}$ finish their LoAdBAlance () call, and all processes in $\mathcal{P}_{i^{*}}$ (including $p$ ) have executed exactly one shared memory access. We distinguish two cases. 
Case 1: In configuration $\boldsymbol{C}, \boldsymbol{p}$ is visible. Let $\mathcal{W}$ be the set of processes in $\mathcal{P}_{i^{*}}$ whose first shared memory access was a write. Note that among all processes in $\mathcal{P}_{i^{*}}$, only processes in $\mathcal{W}$ could have changed the value of a shared register during $H^{\prime}$ (all SC operation must have failed, because they weren't preceded by a LL). Hence, since $p$ is visible in $C, p \in \mathcal{W}$. We let all processes in $\mathcal{W}$, including $p$, take steps in a round-robin way, ordered by their IDs. Since no process in $\mathcal{W}$ can ever see a process not in $\mathcal{P}_{i^{*}}$, all those processes eventually make progress and finish their FETCH\&INC() call. When a process finishes its FETCH\&INC() call, it is stopped and the scheduling continues with the remaining processes in $\mathcal{W}$, until all processes are stopped.

Case 2: In configuration $C, \boldsymbol{p}$ is not visible. Although $p$ is not visible in configuration $C$, it is possible that some processes see $p$ during $H^{\prime}$. This can happen, for example, if $p$ writes to a register $R$, then some process $q$ reads $R$, and finally a third process writes to $R$, overwriting whatever $p$ has written. Let $\mathcal{S}$ be the set that contains $p$ and all processes $q \neq p$ that see $p$ during $H^{\prime}$. We let all processes in $\mathcal{P}_{i^{*}}-\mathcal{S}$ take steps in a round-robin way, ordered by their IDs. Since each process $q \in \mathcal{S}-\{p\}$ sees $p$ when $q$ executes its first shared memory operation, that operation must be either a LL, a read, or a failed SC. Hence, no process in $\mathcal{S}$ is visible in configuration $C$ or has been seen by a process in $\mathcal{P}_{i^{*}}-\mathcal{S}$. Thus, all processes in $\mathcal{P}_{i^{*}}-\mathcal{S}$ eventually make progress and finish their FETCH\&INC() call when scheduled in a round-robin fashion. When this happens for a process, that process is stopped, and the scheduling continues with the other processes, until all processes are stopped. Finally, we let $p$ run until it has finished its FETCH\&INC() call. (Since no other process $q \in \mathcal{S}$ is visible, and $p$ has not seen a process from $\mathcal{S}, p$ 's FETCH\&INC() call will eventually terminate.)

\subsection{Analysis}

For our analysis we assume that $n$ is a perfect square and let $m=\sqrt{n}$. Later in the analysis, we will fix a sequence of coin flips in such a way that each process gets the same coin flips during every run of the algorithm, no matter what the scheduling is. Let $\tilde{c}$ denote such a sequence of coin flips. (Note that $\tilde{c}$ is not the same as the coin-flip vectors $\vec{c}$ used in previous sections.) However, for any fixed adversary $\mathcal{A}$ and a fixed process $p$, each coin-flip sequence $\tilde{c}$ uniquely determines a random history $H$ and we can choose $\tilde{c}$ uniformly at random to determine $X_{\mathcal{A}, p}=X_{\mathcal{A}, p}(\tilde{c})$.

For the analysis, we will let the adversary $\mathcal{A}_{p}$ do the scheduling in order to try to "fool" process $p$. Since we don't know which adversary is the best for the purpose of a lower bound, we choose $p \in \mathcal{P}$ at random. To emphasize that $p, \mathcal{A}$ and $\tilde{c}$ are random (although there will be a dependence between $p$ and $\mathcal{A}$ ), we now denote $X_{\mathcal{A}, p}(\tilde{c})$ by the random variable $X=X(\mathcal{A}, p, \tilde{c})$. We prove the following:

Lemma 5.5. For $\tilde{c}$ and $p \in \mathcal{P}$ chosen uniformly at random,

$$
\mathrm{E}_{p, \tilde{c}}\left[X\left(\mathcal{A}_{p}, p, \tilde{c}\right)\right]=\Omega(\sqrt{n}) .
$$

By averaging over all processes $p \in \mathcal{P}$, there exists a process $p$ such that for random $\tilde{c}$

$$
\mathrm{E}_{\tilde{c}}\left[X\left(\mathcal{A}_{p}, p, \tilde{c}\right]=\Omega(\sqrt{n}) .\right.
$$

Since

$$
\Phi\left(\mathcal{A}_{p}\right)=\max _{q \in \mathcal{P}}\left(\mathrm{E}_{\tilde{c}}\left[X\left(\mathcal{A}_{p}, q, \tilde{c}\right)\right]\right) \geq \mathrm{E}_{\tilde{c}}\left[X\left(\mathcal{A}_{p}, p, \tilde{c}\right)\right]
$$


Theorem 5.3 follows immediately. Thus, it suffices to prove Lemma 5.5.

In order to prove the lemma, we first consider an arbitrarily fixed coin-flip sequence $\tilde{c}$, and we choose only $p \in \mathcal{P}$ uniformly at random. Let $H=H(p)$ be the random history obtained if adversary $\mathcal{A}_{p}$ schedules a run of LoadBalance () , for the fixed coin flips $\tilde{c}$. By construction of $\mathcal{A}_{p}$, during $H$ process $p$ 's FETCH\&INC() call returns. We write $L=L(p)$ for the random variable that denotes the return value of that FETCH\&INC()-call. (Note that the value of $X$ can be 0 even if $L>0$, because in $H$ contention might exceed $K_{\max }$.) Since $\tilde{c}$ is fixed, each process gets the same results from its coin-flips, independently of the scheduling, and thus independently of the choice of $p$. Hence, the set $\mathcal{P}_{i}, 0 \leq i \leq m$, of processes that choose counter $F_{i}$ is fixed, too. Moreover, since we choose $p \in \mathcal{P}$ at random, the counter chosen by $p$ is determined by which set $\mathcal{P}_{i}$ process $p$ is taken from.

In the following, we analyze the expectation of $X$ conditioned under the event that $p$ is in $\mathcal{P}_{j}$ for an arbitrary index $j$. More precisely, we show the following:

Lemma 5.6. For any fixed $\tilde{c}$, any $0 \leq j<m$, and for $p \in \mathcal{P}_{j}$ chosen uniformly at random,

$$
\mathrm{E}[L]=\Omega\left(\left|\mathcal{P}_{j}\right|\right) .
$$

For the ease of notation, we prove the statement for $j=0$. Analogous arguments prove the statement for an arbitrary choice of $j$, and so we lose no generality.

Let $H^{\prime}=H^{\prime}(p)$ be the prefix of $H$ that ends when configuration $C=C(p)$ is reached (i.e., when all processes in $\mathcal{P}_{0}$ are stalled after they have executed their first shared memory access), and let $H^{\prime \prime}=H^{\prime \prime}(p)$ the suffix such that $H=H^{\prime} \circ H^{\prime \prime}$. Let $o p_{q}$ be the shared memory operation process $q \in \mathcal{P}$ performs during $H^{\prime}$. Let $\mathcal{V}$ be the set of processes in $\mathcal{P}_{0}$, that are visible in configuration $C$ and whose first shared memory operation during $H^{\prime}$ is not a write to a register that was previously written by some other process. I.e., the operation $o p_{q}$ of a process $q \in \mathcal{V}$ is a write to some register $R$, such that no other process $q^{\prime}$ writes to $R$ during $H^{\prime}$.

Let $\mathcal{E}$ be the event that $p$ is visible in configuration $C$. Since $p$ is the first process to execute a shared memory access, $\mathcal{E}$ occurs if and only if $p \in \mathcal{V}$. Note that if event $\mathcal{E}$ occurs, then the adversary acts as described under Case 1, otherwise as in Case 2. In the following we give lower bounds on the conditional expectation of $L$ for both cases.

\subsubsection{Analysis of Case 1}

Claim 5.7. The set $\mathcal{V}$ is independent of $p$, and $\mathrm{E}[L \mid \mathcal{E}] \geq(|\mathcal{V}|-1) / 2$.

Proof. Recall that in Case 1 we assume that event $\mathcal{E}$ has occurred, and so $p \in \mathcal{V}$. On the other hand, process $q$ is in $\mathcal{V}$ if and only if $o p_{q}$ is a write to some register $R_{q}$ and no other process writes to $R_{q}$ during $H^{\prime}$. Whether a process writes in the first step, and if so, to which register it writes, depends only on $q$ 's coin-flips, i.e., on $\tilde{c}$, but not on the choice of $p$. Thus, $\mathcal{V}$ is independent of $p$.

Now let $C_{R}=C_{R}(p)$ be the (random) configuration of the shared memory at the end of $H^{\prime}$. (Note that process states are not captured by $C_{R}$.) Note that the position in $H^{\prime}$ of the event where a process $q \in \mathcal{V}$ executes its shared memory operation $o p_{q}$ on register $R_{q}$ has no effect on the configuration $C_{R}$ because $q$ is the only process in $\mathcal{V}$ writing to $R_{q}$. Also, given that $p \in \mathcal{V}$, in $H^{\prime}$ the relative order of steps by processes that are not in $\mathcal{V}$ is independent of $p$. Hence, given that 
$p \in \mathcal{V}$, the register configuration $C_{R}$ is independent of $p$. Formally,

$$
\forall q, q^{\prime} \in \mathcal{V}: C_{R}(q)=C_{R}\left(q^{\prime}\right)
$$

Recall that $\mathcal{W}$ is the set of processes in $\mathcal{P}_{0}$ whose first shared-memory step is a write. Clearly, a process in $\mathcal{W}$ cannot see any other process during $H^{\prime}$, because it performs no more steps after its write. Hence, given $p \in \mathcal{V} \subseteq \mathcal{W}$, what processes in $\mathcal{W}$ observe during $H^{\prime}$ is independent of $p$. Thus,

$$
\forall q, q^{\prime} \in \mathcal{V}: H^{\prime}(q) \sim_{\mathcal{W}} H^{\prime}\left(q^{\prime}\right)
$$

Hence, at the end of $H^{\prime}$ the state of a processor in $\mathcal{W}$ is independent of the choice of $p \in \mathcal{V}$. From this and (5),

$$
\forall q, q^{\prime} \in \mathcal{V}: C(q) \sim_{\mathcal{W}} C\left(q^{\prime}\right) .
$$

In the second part of the scheduling by process $\mathcal{A}_{p}$, which determines $H^{\prime \prime}$, only processes in $\mathcal{W}$ get scheduled in a round-robin way, ordered by their IDs. Given $p \in \mathcal{V} \subseteq \mathcal{W}$, that scheduling is independent of $p$. Since, given $p \in \mathcal{V}$, processes in $\mathcal{W}$ cannot distinguish between the possible configurations $C$ obtained at the end of $H^{\prime}$, they cannot distinguish between the histories $H^{\prime \prime}(p)$, $p \in \mathcal{V}$, either. I.e.,

$$
\forall q, q^{\prime} \in \mathcal{V}: H^{\prime \prime}(q) \sim \mathcal{W} H^{\prime \prime}\left(q^{\prime}\right)
$$

From this and (6), we get

$$
\forall q, q^{\prime} \in \mathcal{V}: H(q) \sim_{\mathcal{W}} H\left(q^{\prime}\right)
$$

As a consequence, given $p \in \mathcal{V}$, the value returned by the FETCH\&INC () of process $q \in \mathcal{V}$ is independent of $p$. Since all processes in $\mathcal{V}$ finish their feTCH\&InC() during $H$, but none of them starts its FETCH\&DEC(), the values processes in $\mathcal{V}$ receive from their FETCH\&INC() calls are all distinct non-negative integers from a (fixed) set $S=\left\{s_{1}, \ldots, s_{|\mathcal{V}|}\right\}$. Thus, given that $p$ is distributed uniformly over $\mathcal{V}$, the return value of $p$ 's FETCH\&INC() is distributed uniformly over $S$. The expectation of that value is at least $(|\mathcal{V}|-1) / 2$.

\subsubsection{Analysis of Case 2}

Claim 5.8. Let $H$ be an arbitrary history of Algorithm 4 (LoadBAlance), and let $P \cup\{p\}$, $p \notin P$, be a set of processes that call $F_{i}$. FETCH\&INC ()$, i \in\{0, \ldots, m-1\}$. Suppose the following hold for $H$ :

(a) the processes in $P \cup\{p\}$ all finish their $F_{i}$.FETCH\&INC(),

(b) no process in $\mathcal{P}$ calls $F_{i}$.FETCH\&DEC () , and

(c) none of the processes in $P$ sees a process in $\bar{P}$.

Then process $p$ 's $F_{i}$. FETCH\&INC() returns a value of at least $|P|$.

Proof. By (a) and (b) all processes in $P \cup\{p\}$ receive distinct values from their FETCH\&InC() calls. By (c) and Observation 5.4, $H$ and $H \mid P$ are indistinguishable to the processes in $P$, and so the return value of a FETCH\&INC() call by a process $q \in P$ is at most $|P|-1$. Hence, the set of FETCH\&INC( $)$ call return values of processes in $P$ is exactly $\{0, \ldots,|P|-1\}$, and so $p$ 's FETCH\&INC() call must return a value of at least $|P|$.

\footnotetext{
${ }^{6}$ With $C_{R}(q)$ we denote the register configuration obtained if the random process $p=q$. Similarly we write $H(q)$, $H^{\prime}(q), H^{\prime \prime}(q)$, and $C(q)$.
} 
Now let $\mathcal{Q}$ be the set of processes in $\mathcal{P}_{0}$ whose first shared-memory access is a read, a LL, or a SC (which fails). Note that $\mathcal{Q}$ is independent of the random choice of $p \in \mathcal{P}_{0}$. Recall that $\mathcal{S}$ is the set that contains $p$ and all processes that see $p$ during $H^{\prime}$. Since the FETCH\&INC() implementation is natural, $\mathcal{S} \subseteq \mathcal{P}_{0}$. Let $S=S(p)=|\mathcal{S}|$.

Claim 5.9. If event $\mathcal{E}$ does not occur, then $L \geq\left|\mathcal{P}_{0}\right|-S \geq\left|\mathcal{P}_{0}\right|-|\mathcal{Q}|$.

Proof. If event $\mathcal{E}$ does not occur, then $p$ is not visible in configuration $C$. By definition of the adversary, during $H^{\prime \prime}$ only processes in $\left(\mathcal{P}_{0}-\mathcal{S}\right) \cup\{p\}$ take steps, and finish their FETCH\&INC () call but don't start their FETCH\&DEC () call.

A process in $\mathcal{S}-\{p\}$ does not "leave a trace" during $H$, i.e., it is never visible: Since it sees $p$ its only operation occurs during $H^{\prime}$, and that operation is either a read, a LL, or a failed SC. In particular, during $H$, no process can see a process in $\mathcal{S}-\{p\}$. In addition, no process $q \in \mathcal{P}_{0}-\mathcal{S}$ sees $p$ during $H$ : It cannot see $p$ during $H^{\prime}$, or else it would be in $\mathcal{S}$, and it cannot see $p$ during $H^{\prime \prime}$, because $p$ remains invisible until $q$ has finished its FETCH\&INC() call and is stopped. Moreover, since the implementation is natural, a process in $\mathcal{P}_{0}$ cannot see a process in $\overline{\mathcal{P}_{0}}$. To conclude, no process in $\mathcal{P}_{0}-\mathcal{S}$ sees any process in $\mathcal{S} \cup \overline{\mathcal{P}_{0}}=\overline{\mathcal{P}_{0}-\mathcal{S}}$. Thus, the conditions of Claim 5.8 are satisfied for $P=\mathcal{P}_{0}-\mathcal{S}$, and so $L \geq\left|\mathcal{P}_{0}-\mathcal{S}\right|=\left|\mathcal{P}_{0}\right|-S$. Because $o p_{q}$ is a read, a LL, or a failed SC for every process $q \in \mathcal{S}$, we have $S \leq|\mathcal{Q}|$ and thus $\left|\mathcal{P}_{0}\right|-S \geq\left|\mathcal{P}_{0}\right|-|\mathcal{Q}|$.

\subsubsection{Putting Things Together}

Proof of Lemma 5.6. W.l.o.g. assume $j=0$. Which operation a process in $\mathcal{P}_{0}$ executes in its first shared memory step is independent of the choice of $p \in \mathcal{P}_{0}$. Thus, since $p$ is uniformly distributed in $\mathcal{P}_{0}$, we have

$$
\operatorname{Prob}(p \in \mathcal{Q})=\frac{|\mathcal{Q}|}{\left|\mathcal{P}_{0}\right|} .
$$

Similarly, since by Claim $5.7|\mathcal{V}|$ is independent of $p$, and since event $\mathcal{E}$ occurs if and only if $p \in \mathcal{V}$,

$$
\operatorname{Prob}(\mathcal{E})=\operatorname{Prob}(p \in \mathcal{V})=\frac{|\mathcal{V}|}{\left|\mathcal{P}_{0}\right|}
$$

Applying Claim 5.7 and (9) we obtain

$$
\mathrm{E}[L \mid \mathcal{E}] \cdot \operatorname{Prob}(\mathcal{E}) \geq \frac{|\mathcal{V}|-1}{2} \cdot \frac{|\mathcal{V}|}{\left|\mathcal{P}_{0}\right|}
$$

If $p \in \mathcal{Q}$, then no process sees $p$ during $H^{\prime}$, i.e., $S=0$. Moreover, if $p \in \mathcal{Q}$, then $p$ is not visible in configuration $C$ and thus $\mathcal{E}$ does not occur. Consequently, by Claim [5.9, $L \geq\left|\mathcal{P}_{0}\right|$, and thus applying (8),

$$
\mathrm{E}[L \mid p \in \mathcal{Q}] \cdot \operatorname{Prob}(p \in \mathcal{Q}) \geq\left|\mathcal{P}_{0}\right| \cdot \frac{|\mathcal{Q}|}{\left|\mathcal{P}_{0}\right|} .
$$

If $p \notin \mathcal{Q} \cup \mathcal{V}$, then $\mathcal{E}$ does not occur, and by Claim 5.9, $L \geq\left|\mathcal{P}_{0}\right|-|\mathcal{Q}|$. Hence, applying the union bound for (8) and (9)

$$
\begin{aligned}
& \mathrm{E}[L \mid p \notin \mathcal{Q} \cup \mathcal{V}] \cdot \operatorname{Prob}(p \notin \mathcal{Q} \cup \mathcal{V}) \geq\left(\left|\mathcal{P}_{0}\right|-|\mathcal{Q}|\right) \cdot\left(1-\frac{|\mathcal{Q}|+|\mathcal{V}|}{\left|\mathcal{P}_{0}\right|}\right) \\
& \geq \frac{\left(\left|\mathcal{P}_{0}\right|-|\mathcal{Q}|-|\mathcal{V}|\right)^{2}}{\left|\mathcal{P}_{0}\right|}
\end{aligned}
$$


Now let

$$
Z=\max \left\{|\mathcal{V}|(|\mathcal{V}|-1) / 2,|\mathcal{Q}| \cdot\left|\mathcal{P}_{0}\right|,\left(\left|\mathcal{P}_{0}\right|-|\mathcal{Q}|-|\mathcal{V}|\right)^{2}\right\}
$$

Since either $|\mathcal{V}| \geq\left|\mathcal{P}_{0}\right| / 3$ or $|\mathcal{Q}| \geq\left|\mathcal{P}_{0}\right| / 3$ or $\left(\left|\mathcal{P}_{0}\right|-|\mathcal{Q}|-|\mathcal{V}|\right) \geq\left|\mathcal{P}_{0}\right| / 3$, we have

$$
Z \geq \min \left\{\frac{\left(\left|\mathcal{P}_{0}\right| / 3\right)\left(\left|\mathcal{P}_{0}\right| / 3-1\right)}{2}, \frac{\left|\mathcal{P}_{0}\right|^{2}}{3},\left(\left|\mathcal{P}_{0}\right| / 3\right)^{2}\right\}=\Omega\left(\left|\mathcal{P}_{0}\right|^{2}\right)
$$

Summing up (10), (11), and (12), we obtain

$$
\mathrm{E}[L] \geq \frac{1}{\left|\mathcal{P}_{0}\right|} \cdot Z=\Omega\left(\left|\mathcal{P}_{0}\right|\right)
$$

We are now ready to prove the main lemma. Up to now, we assumed that the coin flips $\tilde{c}$ are fixed. In the following we have to consider a random choice of $\tilde{c} \in \Omega^{\infty}$, and a random choice of $p \in \mathcal{P}$. We use the fact that the number of processes, $\left|\mathcal{P}_{i}\right|$, that choose a counter object $i$ is highly concentrated around the expectation, $n / m=\sqrt{n}$. Consequently it is unlikely that there is an index $i$ such that $\left|\mathcal{P}_{i}\right|$ is much larger or smaller than $\sqrt{n}$.

Claim 5.10. Let $\mu=n / m$ and $\delta>0$ an arbitrarily small constant. For a randomly chosen $\tilde{c}$, with probability $1-o(1)$ it is true that

$$
\forall 0 \leq i<m:(1-\delta) \mu<\left|\mathcal{P}_{i}\right|<(1+\delta) \mu .
$$

Proof. First consider an arbitrary index $i \in\{0, \ldots, m-1\}$, and let $Y_{i}=\left|\mathcal{P}_{i}\right|$. Since each process $q \in \mathcal{P}$ independently chooses an index $i_{q} \in\{0, \ldots, m-1\}$, the distribution of the random variable $Y_{i}$ is identical to that of the binomial random variable $B(n, 1 / m)$. From Chernoff Bounds and $\mu=n / m=\sqrt{n}$ we obtain

$$
\operatorname{Prob}\left(Y_{i} \leq(1-\delta) \mu \vee Y_{i} \geq(1+\delta) \mu\right) \leq 2 \cdot e^{-\Omega(\mu)}=e^{-\Omega(\sqrt{n})} .
$$

The claim now follows immediately from summing up this probability bound for $Y_{0}, \ldots, Y_{m-1}$.

Proof of Lemma 5.5. Choose $\tilde{c}$ and $p$ uniformly at random and consider the history $H=H\left(\mathcal{A}_{p}, p, \tilde{c}\right)$ obtained by a scheduling of the adversary $A_{p}$. Let $\Gamma$ denote the event that

$$
(1-\delta) m<\left|P_{0}\right|, \ldots,\left|\mathcal{P}_{m-1}\right|<(1+\delta) m .
$$

Note that whether or not $\Gamma$ occurs depends only on $\tilde{c}$ and not on $p$.

Let $L=L\left(\mathcal{A}_{p}, p, \tilde{c}\right)$ denote the return value of $p$ 's FETCH\&INC() call. By Lemma 5.6 and since $\Gamma$ is independent of $p$,

$$
\mathrm{E}[L \mid \Gamma]=\Omega(m)
$$

By definition of $\mathcal{A}_{p}$, if $p \in P_{j}$, then at any point there is at most one process active that is not in $\mathcal{P}_{j}$. Hence, if $\Gamma$ occurs, then the maximum point contention, $K$, satisfies

$$
K \leq \max _{0 \leq i<m}\left|\mathcal{P}_{i}\right|+1<(1+\delta) m+1
$$

and since $K$ is an integer, $K \leq\lceil(1+\delta) m\rceil=K_{\max }$. Hence, given that $\Gamma$ occurs, $X\left(\mathcal{A}_{p}, p, \tilde{c}\right)=L$ and so applying Claim 5.10 and (13),

$$
\mathrm{E}\left[X\left(\mathcal{A}_{p}, p, \tilde{c}\right)\right] \geq \mathrm{E}\left[X\left(\mathcal{A}_{p}, p, \tilde{c}\right) \mid \Gamma\right] \cdot \operatorname{Prob}(\Gamma)=\mathrm{E}[L \mid \Gamma] \cdot(1-o(1))=\Omega(m) .
$$


Acknowledgments We are grateful to the anonymous referees of STOC 2011 for their comments. Thanks also to Faith Ellen for her detailed feedback. 


\section{References}

$\left[\mathrm{AAD}^{+} 93\right]$ Y. Afek, H. Attiya, D. Dolev, E. Gafni, M. Merritt, and N. Shavit. Atomic snapsots of shared memory. J. ACM, 40(4):873-890, 1993.

[Abr88] Karl R. Abrahamson. On achieving consensus using a shared memory. In PODC '88: Proc. of the 6th annual ACM symposium on Principles of distributed computing, pages 291-302, 1988.

[AG88] James H. Anderson and Mohamed G. Gouda. Atomic semantics of nonatomic programs. Inf. Process. Lett., 28(2):99-103, 1988.

[Asp03] James Aspnes. Randomized protocols for asynchronous consensus. Distributed Computing, 16:165-175, 2003.

[CIL87] Benny Chor, Amos Israeli, and Ming Li. On processor coordination using asynchronous hardware. In PODC '8\%: Proc. of the 6th annual ACM symposium on Principles of distributed computing, pages 86-97, 1987.

[GHHW07] W. Golab, V. Hadzilacos, D. Hendler, and P. Woelfel. Constant-rmr implementations of cas and other synchronization primitives using read and write operations. In PODC '07: Proc. of the 26th annual ACM symposium on Principles of distributed computing, pages $0-0,2007$.

[Gol10] W. Golab. Constant-RMR Implementations of CAS and Other Synchronization Primitives Using Read and Write Operations. PhD thesis, University of Toronto, 2010.

[Her91] M. Herlihy. Wait-free synchronization. ACM TOPLAS, 13(1), January 1991.

[HLM03] M. Herlihy, V. Luchangco, and M. Moir. Obstruction-free synchronization: Doubleended queues as an example. In ICDCS '03: Proc. of the 23rd International Conference on Distributed Computing Systems, page 522, Washington, DC, USA, 2003. IEEE Computer Society.

[HS08] M. Herlihy and N. Shavit. The Art of Multiprocessor Programming. Morgan Kaufmann Publishers, first edition, 2008.

[HW90] M. Herlihy and J. M. Wing. Linearizability: A correctness condition for concurrent objects. ACM TOPLAS, 12(3):463-492, July 1990.

[IL93] A. Israeli and M. Li. Bounded time-stamps. Distributed Computing, 6(4):205-209, 1993.

[Lyn96] Nancy Lynch. Distributed Algorithms. Morgan Kaufman, 1996.

[VA86] P. Vitanyi and B. Awerbuch. Atomic shared register access by asynchronous hardware. In Proc. of the 27th Annual Symposium on Foundations of Comupter Science, pages 233-243. IEEE, 1986.

[Vid88] K. Vidyasankar. Converting lamport's regular register to atomic register. Information Processing Letters, 28(6):287-290, August 1988. 
[YA95] J.-H. Yang and J. Anderson. A fast, scalable mutual exclusion algorithm. Distributed Computing, 9(1):51-60, August 1995. 


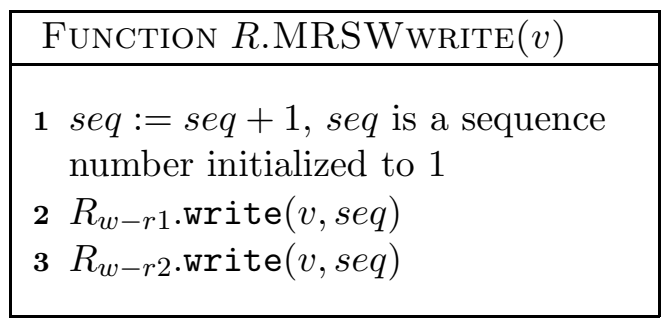

\begin{tabular}{|ll|}
\hline FUNCTION $R$. MRSWREAD \\
\hline $\mathbf{1}$ & code for process $r_{i}, i \in\{1,2\}$ \\
$\mathbf{2}$ & $v[0], s[0]:=R_{w-r i} \cdot$ read \\
$\mathbf{3}$ & $v[1], s[1]:=R_{r 1-r i} \cdot$ read \\
$\mathbf{4}$ & $v[2], s[2]:=R_{r 2-R i} \cdot$ read \\
$\mathbf{5}$ & let $j$ be such that $s[j]=\max \{s[0], s[1], s[2]\}$ \\
$\mathbf{6}$ & $R_{r i-r 1} \cdot$ write $(v[j], \operatorname{seq}[j])$ \\
$\mathbf{7}$ & $R_{r i-r 2} \cdot \operatorname{write}(v[j], \operatorname{seq}[j])$ \\
$\mathbf{8}$ & return $v[j]$ \\
\hline
\end{tabular}

Figure 5: Linearizable Implementation of MRSW Registers from SRSW Registers

\section{A Additional Examples}

When, in a randomized distributed algorithm, atomic operations are replaced by their linearizable implementations, the scheduler's power can increase. Even when the power of the scheduler of the implemented algorithm is constrained significantly from its power in the atomic model, the scheduler can still create a probability distribution of computations that is dramatically different from what is attainable in the atomic case.

The following are various additional examples of this phenomenon.

Implementing multi-reader/single-writer registers from single-reader/single-writer registers. Let $R$ be a two-reader atomic register initialized to 0 , accessed by writer $w$, and readers $r_{1}$ and $r_{2}$ that are executing the code:

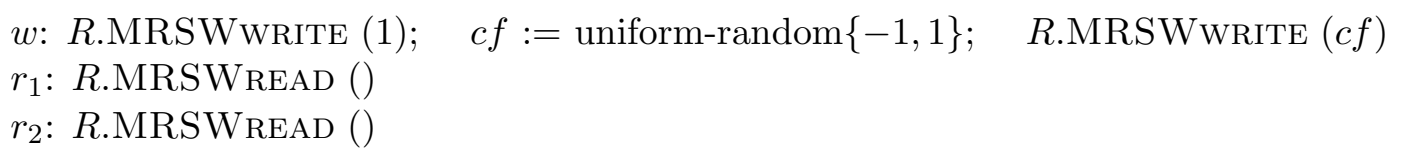

Suppose the strong adversary is trying to minimize the value of $r_{1}$ 's MRSWREAD. If $R$ is an atomic register, then the adversary's best strategy is to have $r_{1}$ execute its MRSWREAD either before or after both of w's MRSWWRITE operations. In either case the expected value of $r_{1}$ 's MRSWREAD is 0.

Now suppose, instead, that $R$ is implemented using the algorithm shown in Figure 5 . This construction uses 6 single-reader/single-writer registers: $R_{w-r 1}, R_{w-r 2}, R_{r 1-r 1}, R_{r 1-r 2}, R_{r 2-r 1}, R_{r 2-r 2}$, where the subscript $x-y$ denotes a register written by $x$ and read by $y$. Each is initialized to the initial value of $R$.

Under this implementation, the adversary's could schedule as follows: First one step of $r_{1}$ : $R_{w-r 1}$.read, which will return 0; then all of w's steps (that is, all of its first MRSWWRITE, its flip and its second MRSWWRITE). If the value of $c f$ is 1 , then the adversary next schedules the rest of $r_{1}$ 's steps, followed by all $r_{2}$ 's steps in its R.MRSWREAD. In this case the implementation of $R$.MRSWREAD by $r_{1}$ returns 0 . If the value of $c f$ is -1 , then the adversary next schedules all $r_{2}$ 's steps in its R.MRSWREAD followed by the remainder of $r_{1}$ 's R.MRSWREAD. In this case, $r_{1}$ will discover the updated value of $R$, when it executes $R_{r 2-r 1}$.read, and the implementation returns -1 . Hence the expected value returned by $r_{1}$ is $-1 / 2$. 


\begin{tabular}{|l|}
\hline FunCTION $Q . \operatorname{EnQ}(v)$ \\
\hline $\mathbf{1}$ pos $:=$ tail.fetch\&inc () \\
$\mathbf{2}$ item $[$ pos $] \cdot \operatorname{write}(v)$ \\
\hline
\end{tabular}

Figure 6: Implementation of the Herlihy-Wing Queue

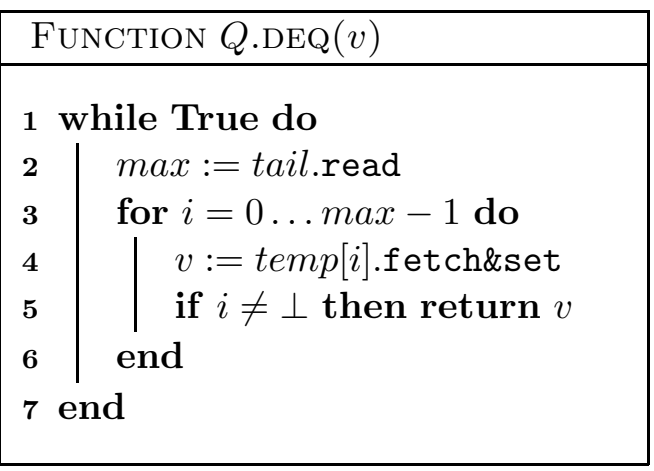

Notice that this schedule is available even to the weak adversary. It needs the coin flip value only after $w$ has completed all its operations. So reducing the power of the adversary from strong to weak does not curtail its power sufficiently to retain the expected behaviour of the algorithm when $R$ is an atomic register.

This algorithm in Figure 5 is the wait-free linearizable implementation of multi-reader/multiwriter multivalued atomic registers from single-reader/single-writer multivalued atomic registers using unbounded sequence numbers due to Vitanyi and Awerbuch ([VA86]) when specialized to two readers and one writer. The increased power of the weak adversary in the implementation over the power of the strong adversary in the atomic case also extends to the case when there is more than one writer.

Implementing a queue with atomic increment objects. Let $Q$ be a queue object, initially empty and accessed by $q_{1}, q_{2}$ and $p$ that are executing the code:

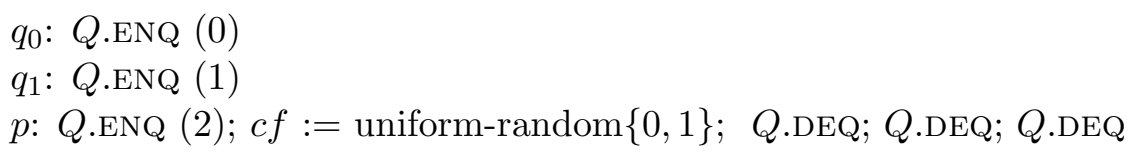

The adversary's goal is to achieve the following:

(a) all of $p$ 's DEQ operations succeed,

(b) the DEQ operation that returns 1 precedes the DEQ operation that returns 2, and

(c) the return value of $p$ 's first DEQ operation equals the result of the flip.

Even if the adversary is strong, in order to achieve (b), it must schedule $q_{1}$ 's ENQ operation before $p$ 's flip operation. Hence, by the time the flip occurs, the decision whether 0 or 1 is in front of the queue has been made, and cannot be changed by the adversary. Therefore, the probability that $p$ 's first DEQ operation returns the value of the flip is at most $1 / 2$.

Herlihy and Wing give a linearizable implementation of a queue using read-modify-write base objects that support the operations fetch\&set, fetch\&inc and read [HW90]. The queue is represented by an unbounded array of items with a tail pointer. The implementation is shown in Figure 6. 

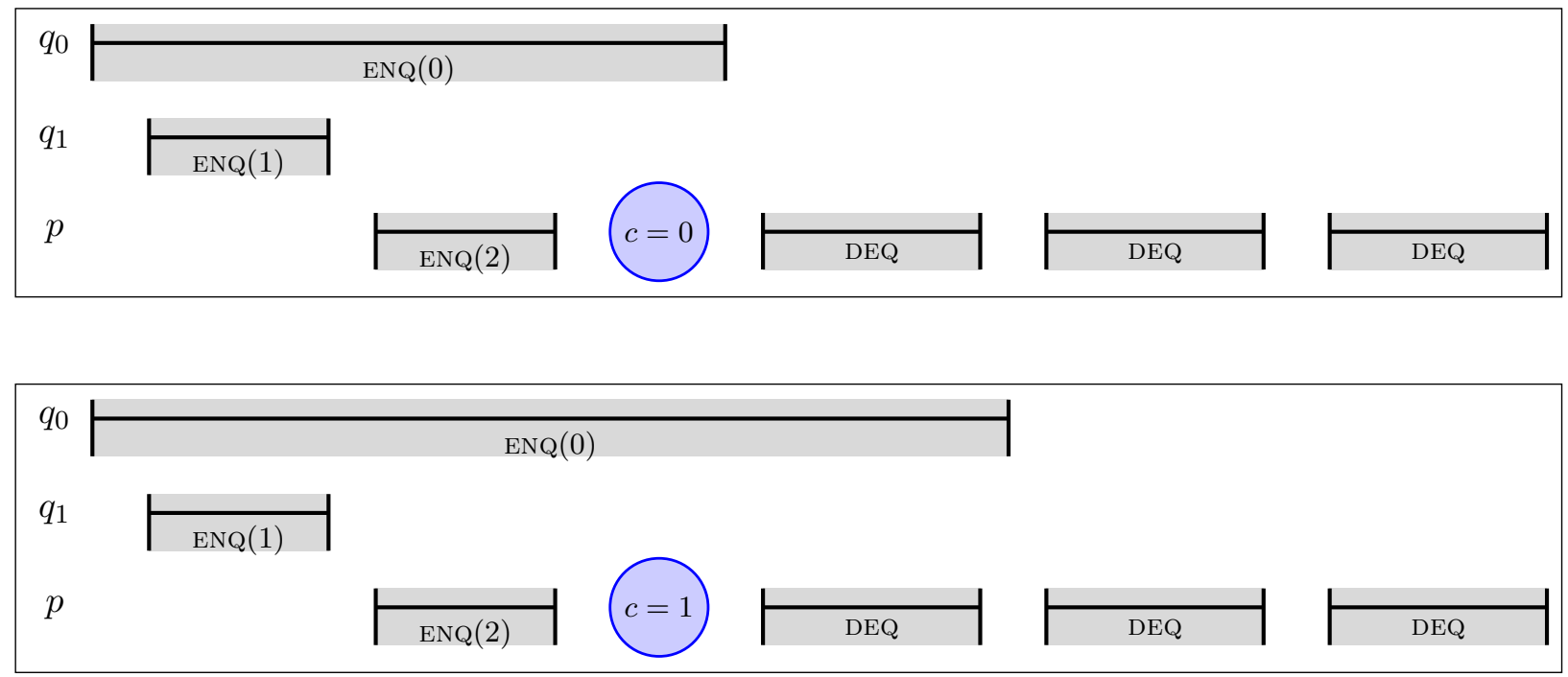

Figure 7: A "bad" scheduling for the queue algorithm.

If the above algorithm uses this queue implementation, then the weak adversary could schedule as shown in Figure 7 . Here, the left bar in the drawing of a queue method call denotes that method call's first shared memory access, and the right bar its last. I.e., the fetch\&inc operations of the ENQ operations occur in the order $q_{0}, q_{1}, p$. And both, $q_{1}$ and $p$ execute their write before the flip happens. If the result of the flip is 0 , then $q_{0}$ writes immediately, before any DEQ operation starts. Therefore, the first DEQ operation will return 0. If the result of the flip is 1 , then $q_{0}$ 's write is delayed until after the first DEQ operation completed. In this case the first DEQ operation will return 1. In either case, the second DEQ operation will return 0 or 1 , and the third will return 2. Hence, even the weak adversary can achieve with probability 1 that (a), (b), and (c) are satisfied. 